\author{
Antonietta Cereghetti Passini \\ Via C. Diener 9, CH-6850 Mendrisio \\ antonietta.cereghetti@gmail.com
}

\title{
Decreti prefettizi pubblicati sulla Gazzetta Ufficiale del Regno d'Italia e l'italianizzazione forzata dei cognomi nelle Nuove Province in generale e nella Provincia dell'Istria in particolare
}

Izvorni znanstveni rad | Original scientific paper UDK 811.163.42'373.2=131.(050)(094.57)"1918/1943"

Primljeno | Received: 22. VIII. 2017.

\section{Sintesi}

Nel primo dopoguerra ebbe inizio un complesso processo di snazionalizzazione delle minoranze presenti da secoli nelle Nuove Province, ossia le terre annesse al Regno d'Italia a seguito dello sfaldamento dell'Impero Asburgico. Nel periodo del governo fascista queste popolazioni divennero oggetto di una marcata attività legislativa che emise decreti prefettizi detti "di riduzione dei cognomi nella forma italiana" al fine d'italianizzare i cognomi di centinaia di migliaia di cittadini.

\begin{abstract}
The aftermath of World War I saw a complex denationalization process taking place which affected the minorities present for many centuries in the Nuove Province annexed to the Kingdom of Italy after the fall of Austria-Hungary. Under the fascist government, these people became the target of an intense legislative activity whereby prefectorial decrees called riduzione dei cognomi nella forma italiana were issued in order to italianize the surnames of hundreds of thousands of citizens.
\end{abstract}

Parole chiave: snazionalizzazione, Nuove Province, Provincia d'Istria, Pola, cognomi, fascismo

Key words: denationalization, Nuove Province, Istria Province, Pula, surnames, fascism 


\section{Introduzione}

Nei due anni successivi alla Grande Guerra, il Trattato di pace di Saint-Germain (10 settembre 1919) e il successivo Trattato di Rapallo (12 novembre 1920) sancirono i confini tra la neoproclamata Repubblica di Austria e, rispettivamente, il neocostituito Regno dei Serbi, Croati e Sloveni e il Regno d'Italia. Quest'ultimo beneficiò del conseguente ridisegnamento geopolitico e riunì nei propri confini i territori delle terre irredente soggette al dominio dell'impero austro ungarico ora dissolto, integrandoli in un primo tempo nella geografia amministrativa italiana secondo la precedente configurazione. Queste terre divennero ben presto oggetto di una riorganizzazione più consona alle ambizioni nazionalistiche delle nuove forze politiche italiane salite al potere nel 1922 .

Con il Trattato di Roma del 27 gennaio 1924 questa riorganizzazione coinvolse anche lo Stato Libero di Fiume: Fiume fu annessa al Regno d'Italia "mentre Sussak, il centro croato contiguo, sarebbe passato alla Jugoslavia". Va ricordato che lo Stato Libero di Fiume, costituito quattro anni prima con il Trattato di Rapallo (articolo 4), era di fatto sotto il diretto controllo politico delle forze fasciste già dal 3 marzo 1922 a seguito di un colpo di Stato fascista ${ }^{2}$.

Con il Regio Decreto (di seguito R.D.) del 18 gennaio 1923, n. 53, vennero istituite la provincia dell'Istria con capoluogo Pola (articolo 1) e quella di Trieste (articolo 2); queste assorbirono una piccola parte dell'antica Contea principesca di Gorizia e Gradisca (articolo 3), la quale cessava di esistere poiché la sua parte restante e maggiore veniva contemporaneamente assegnata alla già esistente provincia di Udine, ridenominata, con lo stesso decreto (articolo 4), provincia del Friuli. Nello stesso giorno di quell'anno il R.D. n. 54 stabiliva l'istituzione della provincia di Zara e tre giorni più tardi, con il R.D. n. 93, nasceva la provincia di Trento comprendente anche il circondario di Bolzano. Quasi un anno dopo fu la volta della provincia del Carnaro con capoluogo Fiume che secondo il Regio decreto-legge (di seguito R.D.L.) n. 213 del 22 febbraio 1924 comprendeva "due circondari costituiti l'uno dalla città di Fiume col territorio annesso al Regno in virtù del R. decreto-legge 22 febbraio 1924, n. 211, e l'altro dall'attuale circondario di Volosca Abbazia, che viene distaccato dalla provincia dell’Istria, eccettuati

1 Marina Cattaruzza, L'Italia e il confine orientale 1866-2006, Bologna 2007, 166 (citando I. J. Lederer).

2 Idem, 165-166. 
i comuni di Castelnuovo e Matteria che vengono aggregati al circondario di Capodistria”. Il processo di riorganizzazione amministrativa si completò con lo scorporamento delle province di Bolzano e Gorizia, rispettivamente dalle province di Trento e di Udine (R.D.L. n. 1 del 2 gennaio 1927).

Durante questa progressiva revisione, vennero create oppure soppresse altre province del Regno, ma il termine Nuove Province veniva riferito "esclusivamente alle Province e ai Comuni redenti e annessi al Regno a seguito della guerra vittoriosa" - come precisato negli Indici generali per l'anno 1923 della Gazzetta Ufficiale del Regno d'Italia (di seguito G.U.), ossia a quelle province e comuni la cui annessione garantì l'unificazione delle Tre Venezie: le Province di Trento e Bolzano (Venezia Tridentina), le Province di Trieste, Istria, Gorizia, Fiume e Zara (Venezia Giulia) nonché i comuni Chiopris Viscone e Tarvisio assorbiti nella Provincia di Udine (facente parte della Venezia Euganea). Parte ora di uno Stato omogeneo, quest'area geografica andava resa italianissima con un minuzioso programma di cancellazione di tutto "quanto non [si] riusciva a classificare come nazionale italiano" . Difficoltà e diffidenza sorte sin dai primi giorni di amministrazione italiana tra i militari e le popolazioni non italiane della Venezia Giulia e Tridentina non solo non vennero meno, bensì crebbero "sotto l'influenza degli italiani del luogo": i "quadri del vecchio partito liberal-nazionale vedevano con sfavore un ampio riconoscimento linguistico delle lingue slovena e croata" e con l'arrivo del governo fascista, l'ostilità si tradusse in un attacco sempre più diretto ai diritti legittimi delle minoranze. Parlando degli slavi giuliani ma la situazione era identica per tutte le etnie non italiane delle Nuove Province - Elio Apih afferma che "la snazionalizzazione fu perseguita attraverso un complesso di misure: distruzione della loro classe dirigente e riduzione numerica del loro gruppo etnico; liquidazione di quanto ancora sussisteva delle loro organizzazioni economiche e culturali; soppressione della scuola slava e della stampa e della lingua slava; italianizzazione dei cognomi; controllo o allontanamento del clero slavo o slavofilo; reazione violenta ad ogni

3 Elio Apih, Trieste, Roma-Bari 1988, 128.

4 Vanni D'Alessio, "Dall'Impero d'Austria al Regno d'Italia. Lingua, stato e nazionalizzazione in Istria" (in seguito: "Dall'Impero d'Austria al Regno d'Italia"), in Una storia balcanica. Fascismo, comunismo e nazionalismo nella Jugoslavia del Novecento, a cura di Lorenzo Bertucelli e Mila Orlić, Verona 2008, 31-71, 56; vedi pure: Hrvoje Mezulić - Roman Jelić, O talijanskoj upravi u Istri i Dalmaciji 1918.-1943. Nasilno potalijančivanje prezimena, imena i mjesta, Zagreb 2005, 19, 132-133.

5 D’Alessio, “Dall'Impero d'Austria al Regno d'Italia”, 57. 
resistenza o tentativo di allusione di queste misure; potenziamento, infine, delle comunità italiane, anche a mezzo di nuovi insediamenti”.

Questa operazione di cancellazione delle diverse etnie presenti da secoli nelle terre da poco annesse venne documentata meticolosamente nella G.U. la quale, coi suoi numerosi decreti e leggi, divenne col tempo "uno degli strumenti principali della presa di possesso italiana [delle Nuove Province]” e rimane tuttora una "fonte affidabile sulla politica di snazionalizzazione del fascismo italiano"7.

A questo riguardo, assai significativo per la nostra indagine, è il R.D.L. n. 17 del 10 gennaio $1926^{8}$, convertito nella legge n. 898 il 24 maggio $1926^{9}$. Questo decreto, suddiviso in tre articoli affiancati dalle istruzioni esposte nel Decreto ministeriale del 5 agosto $1926^{10}$, avrebbe dato il via ufficiale all'italianizzazione forzata dei cognomi non italiani nella provincia di Trento.

Larticolo 1 imponeva alle famiglie della Provincia di Trento "che portano un cognome originario italiano o latino tradotto in altre lingue o deformato con grafia straniera o con l'aggiunta di suffisso straniero [di] riassumere il cognome originario nelle forme originarie. Saranno egualmente ricondotti alla forma italiana i cognomi di origine toponomastica, derivanti da luoghi, i cui nomi erano stati tradotti in altra lingua, o deformati con grafia straniera, e altresì i predicati nobiliari tradotti o ridotti in forma straniera. La restituzione in forma italiana sarà pronunciata con decreto del Prefetto della Provincia, che sarà notificato agli interessati, pubblicato nella Gazzetta Ufficiale del Regno ed annotato nei registri dello stato civile. Chiunque, dopo la restituzione avvenuta, fa uso del cognome o del predicato nobiliare nella forma straniera, è punito con la multa da L. 500 a L. 5000.”

Larticolo 2 garantiva la possibilità "di ridurre in forma italiana con decreto del Prefetto i cognomi stranieri o di origine straniera, quando vi sia la richiesta dell'interessato."

Infine, con l'articolo 3, si rendeva possibile l'estensione degli artt. 1 e 2 ad altre province del Regno, estensione che sarebbe stata decretata con R.D.

6 Elio Apih, Italia, fascismo e antifascismo nella Venezia Giulia (1918-1943) (in seguito: Italia, fascismo e antifascismo), Bari 1966, 274; vedi pure Josip Bratulić - Petar Šimunović nella prefazione del Prezimena $i$ naselja u Istri. Narodnosna statistika u godini oslobođenja, prema Cadastre national de l'Istrie i drugim izvorima priredili Josip Bratulić i Petar Šimunović, Istra kroz stoljeća VI/35, Pula-Rijeka 1985-1986, I, 11.

7 Rolf Wörsdörfer, Il confine orientale, Italia e Jugoslavia dal 1915 al 1955 (in seguito: Il confine orientale), Bologna 2009, 127.

8 Gazzetta Ufficiale dell'Regno d'Italia (in seguito: G.U.), n. 11 del 15 gennaio 1926, 135.

9 G.U., n. 130 del 7 giugno 1926, 2290-2291.

10 G.U., n. 197 del 25 agosto 1926, 3718-3719. 
n. 494 del 7 aprile $1927^{11}$ poco più di un anno dopo con validità per tutto il territorio delle Nuove Province.

Le nuove disposizioni di legge impressero una nuova direzione al mutamento dei cognomi non ritenuti canonici, un processo iniziato nel 1919 - facendo riferimento a una vecchia legge austriaca - e proseguito durante tutti gli anni seguenti nei comuni delle province interessate ${ }^{12}$ mentre nello Stato di Fiume, anticipatore di "tutto quanto doveva accadere in Italia dopo la conquista fascista”"13, il Consiglio Nazionale il 4 aprile 1919 promulgò una legge che autorizzava la correzione dei cognomi “d'origine italiana” come pure il cambiamento e la modifica dei cognomi stranieri ${ }^{14}$.

Facendo riferimento ai nuovi articoli di legge i prefetti di tutte le province nominarono commissioni speciali con l'incarico di compilare l'elenco dei cognomi soggetti a rettifica e di determinarne altresì il cognome sostitutivo. Gli elenchi prodotti poggiavano sulla teoria "che tutti o gran parte dei cognomi negli ex territori asburgici avevano origini italiane ed erano stati germanizzati o slavizzati nell'Ottocento da parroci tedeschi o slavi”'15. La semplice iscrizione nei registri dello stato civile di cognomi e nomi stranieri in base alla pronuncia e all'ortografia italiane, una pratica coltivata "sin dai tempi della monarchia asburgica dall'amministrazione pubblica nei comuni diretti da Italiani” ${ }^{16}$, diveniva pertanto insufficiente perché nelle Nuove Province "dove l'italianità era tornata in auge dopo secoli di dominazione straniera non doveva più esserci spazio per nomi stranieri"17. I decreti prefettizi "che cominciarono a fioccare" nelle province interessate avrebbero ulteriormente dimostrato che "era in corso un'italianizzazione forzata di cognomi slavi che erano tali sin dai tempi più lontani”"18.

11 G.U., n. 93 del 22 aprile $1927,1690$.

12 D’Alessio, “Dall'Impero d'Austria al Regno d'Italia”, 63; Hrvoje Mezulić, Fašizam, krstitelj i palikuća, Pazin 1997, 56; Paolo Parovel, Izbrisani identitet, Pazin-Poreč-Pula 1993, 24-25; Miro Tasso, "Fascismo e cognomi: italianizzazioni coatte nella provincia di Trieste" (in seguito: "Fascismo e cognomi”), in Lo spettacolo delle parole. Studi di storia linguistica e di onomastica in ricordo di Sergio Raffaelli, a cura di Enzo Caffarelli e Massimo Fanfani, Quaderni Italiani di RIOn, 3, Roma 2011, 311-312.

13 Gaetano Salvemini, Le origini del fascismo in Italia, Lezioni di Harvard, Milano 1979, 236.

14 Daniel Patafta, "Promjene u nacionalnoj strukturi stanovništva grada Rijeke od 1918. do 1924. godine", Časopis za suvremenu povijest, 36, 2, 2004, 690, citando "Bollettino ufficiale del Consiglio Nazionale", La Bilancia, n. 72 del 29 marzo 1919, 4.

15 Wörsdörfer, Il confine orientale, 130.

16 Lavo Čermelj, Sloveni e croati in Italia tra le due guerre (in seguito: Sloveni e croati), Trieste 1974, 141.

17 Wörsdörfer, Il confine orientale, 127.

18 Čermelj, Sloveni e croati, 145. 
Il numero di persone soggette al cambiamento è stimato in almeno $500.000^{19}$, ma, contrariamente a quanto imposto dall'articolo 1 del R.D.L. 17, non tutti i decreti emanati sarebbero stati pubblicati nella G.U. così che il numero dei decreti effettivamente pubblicati e qui analizzati, pur rappresentando una sottostima delle cifre reali, è nondimeno tale da legittimare la definizione di "onomasticidio di Stato" ${ }^{20}$. Questi documenti ufficiali testimoniano altresì della presenza continua e profonda delle diverse etnie nelle Nuove province così come rivelano la vasta gamma di cognomi violati, storpiati o artefatti e in parte condannati alla definitiva estinzione per dare spazio a cognomi che le nuove generazioni portano ignorandone le vere origini storiche.

\section{Numero di decreti prefettizi pubblicati}

Le rilevazioni statistiche e le rispettive tabulazioni qui esposte fanno capo ai 49.310 decreti pubblicati sulla G.U. - "la più importante pubblicazione ufficiale dello Stato italiano” per usare la definizione data nel 1932 dal giurista Guido Zanobini nella rispettiva voce dell'Enciclopedia Italiana. Il membro dell'Accademia dei Lincei proseguiva il suo articolo precisando che la G.U. "è divisa in due parti formate da fogli separati e distinte con separata numerazione. Nella parte prima si pubblicano: a) tutte le leggi; b) i decreti reali che debbono essere inseriti nella Raccolta ufficiale delle leggi e decreti, cioè tutti, esclusi solo quelli che riguardano enti e persone singole e quelli la cui pubblicità possa nuocere agl'interessi dello Stato; c) anche gli altri decreti reali e ministeriali di carattere speciale, quando tale pubblicazione sia richiesta dal ministro proponente d'accordo col ministro guardasigilli; d) tutte le nominali e le circolari esplicative di provvedimenti legislativi, eccetto quelle di carattere riservato; e) le disposizioni emanate dalla Presidenza del consiglio dei ministri, dalla Presidenza delle due camere e dal ministero della Real Casa. (...) Fra gli atti pubblicati nella prima parte debbono essere specialmente considerati le leggi e i decreti d'interesse generale, per i quali è prescritta altresì l'inserzione nella Raccolta ufficiale delle leggi e dei decreti del Regno". ${ }^{1}$

19 Ibidem; Parovel, Izbrisani identitet, 28.

20 Miro Tasso, Un onomasticidio di Stato, Trieste 2010.

21 Guido Zanobini, “Gazzetta ufficiale”, Enciclopedia Italiana, 1932 (http://www.treccani.it, visto maggio 2016). 
I decreti prefettizi di mutazione dei cognomi venivano pubblicati nella rubrica "Leggi e decreti” sottorubrica "Decreti prefettizi”, sotto il titolo "Riduzione di cognomi nella forma italiana”. Eppure quest'ultimo era ingannevolmente impreciso poiché i decreti detti "di riduzione” (emessi quando il cognome veniva accordato su richiesta del titolare) erano distinti da quelli “di restituzione”, emessi quando il cambiamento era imposto dall'autorità ${ }^{22}$. Dato il loro elevato numero, i decreti finirono per monopolizzare la G.U. negli anni successivi, a testimonianza della frenesia che animava il processo di snazionalizzazione.

Il primo decreto prefettizio fu pubblicato il 13 ottobre $1927^{23}$ mentre l'ultimo risale al 23 luglio $1937^{24}$ con un totale di ben 49.310 decreti pubblicati. Moltiplicando questa cifra con una media di 4,17 persone per decreto - desunta dal conteggio esatto effettuato per la Provincia dell'Istria - si può stimare che il numero di soggetti toccato dalle nuove leggi ammonti oltre 205.000.

La Tabella 1 riporta tutti i decreti pubblicati secondo la provincia di provenienza e l'anno di pubblicazione. La quasi totalità dei decreti fu pronunciata dai prefetti delle Nuove Province, eccezion fatta per due decreti emessi dalla Prefettura di Padova, due dalla Prefettura di Piacenza e uno dalla Prefettura di Grosseto. La più coinvolta risulta essere la Prefettura di Trieste (44,87\%), seguita dalla Prefettura di Pola $(34,10 \%)$ e dalla Prefettura di Gorizia (10,20 \%) mentre il restante $10,83 \%$ riguarda le prefetture di Bolzano, Fiume (o Carnaro), Zara, Trento e Udine.

Nella statistica non sono compresi sei decreti dedicati alla rettifica degli errori presenti nei decreti già pubblicati, un decreto concernente la revoca di una forma di cognome con imposizione di una alternativa e infine un decreto di revoca concernente un decreto che però non risulta pubblicato. Va precisato che nella documentazione consultata nel sito dell'Automazione della Gazzetta Ufficiale Storica (http://augusto.digitpa.gov.it), oltre a qualche rara pagina, mancanoanchediecinumericompletidella G.U.,parteunoeprecisamente: 1929 il n. 270,1930 il n. 155, 1931 il n. 43, 1934 i n. 101 e 113, 1935 il n. 273, 1936 i n. 82,112 e 136,1937 il n. 58.

22 Tasso, "Fascismo e cognomi", 312; Čermelj, Sloveni e croati, 141-149; Parovel, Izbrisani identitet, 25.

23 G.U., n. 237 del 13 ottobre 1927, 4070.

24 G.U., n. 169 del 23 luglio 1937, 2782. 


\begin{tabular}{|c|c|c|c|c|c|c|c|c|c|c|c|}
\hline \multirow{2}{*}{ Anno } & \multirow{2}{*}{$\begin{array}{c}\text { Totale } \\
\text { decreti } \\
\text { pubblicati }\end{array}$} & \multirow{2}{*}{$\begin{array}{l}\% \text { per } \\
\text { anno }\end{array}$} & \multicolumn{5}{|c|}{ Venezia Giulia } & \multicolumn{2}{|c|}{$\begin{array}{c}\text { Venezia } \\
\text { Tridentina }\end{array}$} & \multirow{2}{*}{$\begin{array}{l}\text { Udine } \\
\text { Udine }\end{array}$} & \multirow{2}{*}{\begin{tabular}{|l} 
Diversi \\
Diversi
\end{tabular}} \\
\hline & & & Trieste & Istria & Gorizia & Fiume & Zara & Trento & Bolzano & & \\
\hline 1927 & 243 & 0,49 & 160 & 0 & 0 & 0 & 0 & 45 & 38 & 0 & 0 \\
\hline 1928 & 4.691 & 9,51 & 3.034 & 1.277 & 0 & 78 & 98 & 92 & 102 & 9 & 1 \\
\hline 1929 & 4.314 & 8,75 & 2.818 & 939 & 0 & 324 & 110 & 102 & 9 & 11 & 1 \\
\hline 1930 & 4.724 & 9,58 & 3.329 & 914 & 0 & 290 & 75 & 90 & 7 & 16 & 3 \\
\hline 1931 & 6.935 & 14,06 & 2.792 & 1.939 & 1.831 & 61 & 244 & 51 & 2 & 15 & 0 \\
\hline 1932 & 5.877 & 11,92 & 1.086 & 2.749 & 1.823 & 91 & 36 & 90 & 0 & 2 & 0 \\
\hline 1933 & 5.170 & 10,49 & 1.550 & 2.586 & 928 & 69 & 21 & 4 & 4 & 8 & 0 \\
\hline 1934 & 4.454 & 9,03 & 2.975 & 1.161 & 223 & 83 & 5 & 0 & 4 & 3 & 0 \\
\hline 1935 & 6.429 & 13,04 & 3.049 & 1.459 & 178 & 313 & 21 & 12 & 1.393 & 4 & 0 \\
\hline 1936 & 5.448 & 11,05 & 349 & 3.791 & 47 & 142 & 14 & 8 & 1.093 & 4 & 0 \\
\hline 1937 & 1.025 & 2,08 & 986 & 0 & 0 & 0 & 0 & 0 & 39 & 0 & 0 \\
\hline Totale & 49.310 & 100,00 & 22.128 & 16.815 & 5.030 & 1.451 & 624 & 494 & 2.691 & 72 & 5 \\
\hline $\begin{array}{l}\% \\
\text { prefett. }\end{array}$ & & 100,00 & 44,87 & 34,10 & 10,20 & 2,94 & 1,27 & 1,00 & 5,46 & 0,15 & 0,01 \\
\hline
\end{tabular}

Tabella 1. Decreti prefettizi pubblicati sulla G.U. secondo anno di pubblicazione e provincia

Secondo Lavo Čermelj l'azione per l'italianizzazione dei cognomi doveva diventare nell'intento delle autorità fasciste un vero e proprio plebiscito. Tutto l'apparato del potere pubblico venne mobilitato: si cercò in tutti i modi di convincere gli abitanti a richiedere "volontariamente" la "restituzione" in forma italiana dei loro cognomi ${ }^{25}$. Il risultato non fu soddisfacente se si considera che ben il $51,5 \%$ dei decreti pubblicati risulta imposto di fronte al 34,0 \% di richieste spontanee (Tabella 2). Del restante 14,5\% non viene fornita alcuna informazione: verosimilmente pure questi cambiamenti furono imposti. Rapportando le percentuali delle richieste al totale dei decreti emessi nelle singole province le più condiscendenti sembrano essere quelle di Udine $(93,1 \%)$ e di Fiume (90,1\%), seguite da quelle di Bolzano (55,1\%), Trieste (54,5 \%), Zara (52,7 \%) e Trento (52,2 \%). Su un totale di 16.815 decreti pubblicati per la provincia dell'Istria ci sono solo 1.262 domande spontanee $(7,5 \%)$ mentre nella provincia di Gorizia tutti i cambiamenti risultano essere imposti.

Va qui sottolineato che la maggior parte degli storici concordano sulla dubbia genuinità di buona parte delle richieste ${ }^{26}$; inoltre, l'assenza dei decreti di revoca (oltre a quello citato sopra) dimostra che la possibilità di veder accolto un ricorso contro il cognome imposto era "puramente fittizia" ${ }^{27}$.

25 Čermelj, Sloveni e croati, 146.

26 Ibidem; Apih, Italia, fascismo e antifascismo, 281; Parovel, Izbrisani identitet, 27; Wörsdörfer, Il confine orientale, 131.

27 Gabriella Klein, La politica linguistica del fascismo, Bologna 1986, 108. 


\begin{tabular}{|l|r|r|r|r|r|r|r|}
\hline Provincia & \multicolumn{1}{c|}{ Totale } & Su richiesta & \multicolumn{1}{c|}{ \% } & \multicolumn{1}{c|}{ Imposti } & \multicolumn{1}{c|}{$\%$} & Non definiti & $\%$ \\
\hline Trieste & $\mathbf{2 2 . 1 2 8}$ & 12.048 & 54,5 & 8.745 & 39,5 & 1.335 & 6,0 \\
Istria & $\mathbf{1 6 . 8 1 5}$ & 1.262 & 7,5 & 11.058 & 65,8 & 4.495 & 26,7 \\
Gorizia & $\mathbf{5 . 0 3 0}$ & 0 & 0,0 & 4.983 & 99,1 & 47 & 0,9 \\
Fiume & $\mathbf{1 . 4 5 1}$ & 1.308 & 90,1 & 1 & 0,1 & 142 & 9,8 \\
Zara & $\mathbf{6 2 4}$ & 329 & 52,7 & 281 & 45,0 & 14 & 2,3 \\
Trento & 494 & 258 & 52,2 & 228 & 46,2 & 8 & 1,6 \\
Bolzano & $\mathbf{2 . 6 9 1}$ & 1.484 & 55,1 & 75 & 2,8 & 1.132 & 42,1 \\
Udine & $\mathbf{7 2}$ & 67 & 93,1 & 1 & 1,4 & 4 & 5,5 \\
Diversi & $\mathbf{5}$ & 5 & 100 & & 0,0 & & 0,0 \\
\hline Totale & 49.310 & $\mathbf{1 6 . 7 6 1}$ & 34,0 & $\mathbf{2 5 . 3 7 2}$ & 51,5 & 7.177 & 14,5 \\
\hline
\end{tabular}

Tabella 2. Decreti prefettizi pubblicati sulla G.U. secondo il criterio richiesto/imposto

\section{Ritmo delle pubblicazioni e peso sullo spazio del foglio ufficiale}

Nonostante il 93,38 \% di tutti i decreti riguardi la Venezia Giulia (Tabella 1), bisogna precisare che i primi 19 decreti - pubblicati fra il 13 ottobre e il 10 novembre 1927 - provenivano dalla Venezia Tridentina. I primi decreti emessi per la Venezia Giulia arrivarono qualche settimana più tardi, il 23 novembre, e interessarono la provincia di Trieste. Lanno successivo avrebbero fatto la loro comparsa i primi decreti dalle province di Udine (23 gennaio), Carnaro (27 febbraio), Zara (1 marzo) ed Istria (21 marzo). All'undici ottobre 1929 risale il primo uso della denominazione "Provincia di Fiume" (alternato con "Provincia di Carnaro") per i decreti emessi dall'omonima prefettura. L’ultima provincia a comparire sarà quella di Gorizia i cui primi 21 decreti saranno pubblicati il 25 aprile 1931.

Nell'anno 1927 furono pubblicati solo 243 decreti, ma a partire dall'anno 1928 il ritmo delle pubblicazioni sarebbe incrementato drasticamente per mantenersi abbondantemente sopra i 4.000 decreti annui per i tre anni successivi. I quasi 7.000 decreti del 1931, anno in cui ebbero inizio le pubblicazioni dalla prefettura di Gorizia, rappresentano il culmine di un processo che si arrestò soltanto nel luglio del 1937, dopo aver sfornato un totale di quasi 50.000 decreti. Questa mole di atti congestionò le capacità di stampa della G.U. e comportò inevitabili ritardi nelle due province più popolose: in quella di Trieste le pubblicazioni ebbero dilazioni fino a tre anni, mentre i ritardi nella provincia dell'Istria lievitarono rapidamente fino a raggiungere i quattro anni.

Lo spazio della G.U. dedicato alle pubblicazioni dei decreti fu esteso sin dall'inizio poiché essi venivano pubblicati in forma integrale, ciascuno 
occupando anche più di un quarto di pagina. Pur con variazioni di contenuto da prefettura a prefettura, ogni decreto riportava, oltre alle formule di richiamo alle relative leggi e alla prefettura di provenienza, anche le voci seguenti: la precisazione se il cambiamento del cognome fosse imposto oppure "accordato" a richiesta; i dati personali del titolare (nome, cognome, paternità, luogo e data di nascita e, se del caso, il titolo); il nuovo cognome da applicare; l'elenco dei famigliari ai quali quello era esteso, indicando il grado di parentela con il titolare e le date di nascita. Convalidava il decreto la sua data d'emissione nonché la firma del prefetto o del suo rappresentante. Eccezion fatta per il periodo iniziale, ogni decreto pubblicato riportava anche il numero del decreto assegnatogli dalla prefettura di provenienza ed il numero d'ordine di redazione. Per talune province compaiono inoltre il luogo di residenza e l'indirizzo esatto del titolare del decreto, la sua professione ed il nome e cognome della madre; è indicata anche la data ed il luogo di nascita di tutti i famigliari e, per mogli, madri e nuore, il nome e il cognome di entrambi i genitori.

Quando nel novembre dell'anno 1935, la G.U. passò a un’impaginazione volta al risparmio di spazio, i decreti prefettizi ne risentirono. Con l'introduzione della spaziatura ridotta fu dimezzata la superficie necessaria per la pubblicazione dei decreti ma dopo soli 211 decreti pubblicati col nuovo approccio, si sarebbe optato per un drastico cambiamento: a partire dall'11 dicembre 1935, infatti, i decreti prefettizi sarebbero stati pubblicati in forma di tabella di otto colonne, a ogni titolare veniva dedicata una sola riga e le informazioni relative ai familiari - ridotte al solo nome e al grado di parentela - venivano relegate in una sola casella. Cosicché per gli ultimi 7.158 decreti manca la parte essenziale all'identificazione delle persone coinvolte.

Nella Tabella 3 vengono comparate le pagine complessive della G.U. parte prima con il numero delle pagine dedicate alla pubblicazione dei decreti. Nei dieci anni interessati dai decreti prefettizi era loro dedicato mediamente il 20,38 \% del totale delle pagine, con punte massime negli anni 1931 e 1932 (29,13\% risp. $27,31 \%)$. Considerata la natura della Gazzetta, l'importanza data alla cancellazione dei segni delle origini di una larga parte della popolazione dei territori annessi diventa inequivocabilmente l'indice di quell'ossessione nazionalistica rammentata dallo storico Klaus Gatterer ${ }^{28}$. 


\begin{tabular}{|c|c|c|c|c|}
\hline Anno & $\begin{array}{c}\text { Numero decreti } \\
\text { prefettizi }\end{array}$ & $\begin{array}{l}\text { Pagine con decreti } \\
\text { prefettizi }\end{array}$ & Totale pagine G.U. & $\begin{array}{l}\% \text { pagine con } \\
\text { decreti prefettizi }\end{array}$ \\
\hline 1927 (dal 02.11 al 31.12) & 243 & 64,50 & 760 & 8,49 \\
\hline 1928 & 4.691 & $1.233,50$ & 6.352 & 19,42 \\
\hline 1929 & 4.314 & $1.154,50$ & 5.813 & 19,86 \\
\hline 1930 & 4.724 & 1.215 & 5.684 & 21,38 \\
\hline 1931 & 6.935 & $1.844,25$ & 6.332 & 29,13 \\
\hline 1932 & 5.877 & 1.599 & 5.856 & 27,31 \\
\hline 1933 & 5.170 & $1.382,50$ & 6.008 & 23,01 \\
\hline 1934 & 4.454 & 1.105 & 5.916 & 18,68 \\
\hline 1935 (dal 01.01 al 21.11) & 5.725 & $1.401,75$ & 5.452 & 25,71 \\
\hline Totale pubbl. dati completi & 42.133 & 11.000 & 48.173 & 22,83 \\
\hline 1935 (dal 22.11 al 31.12) & 704 & 26,75 & 404 & 6,62 \\
\hline 1936 & 5.448 & 187 & 3.808 & 4,91 \\
\hline 1937 (dal 01.01 al 23.07) & 1.025 & 30,25 & 2.788 & 1,09 \\
\hline Totale & 49.310 & 11.244 & 55.173 & 20,38 \\
\hline
\end{tabular}

Tabella 3. Spazio delle G.U. - parte prima riservato ai decreti prefettizi negli anni di pubblicazione

Lassenza di pubblicazioni dei decreti prefettizi a partire dagli ultimi anni Trenta non era sicuramente dovuta alla mancanza di cognomi da "correggere”. Nella sola Prefettura di Pola, per esempio, vi erano ben 9.986 pratiche non convalidate da pubblicazione ${ }^{29}$. Alla fine degli anni Trenta per l'amministrazione italiana, sollecitata dalle insistenti richieste dei vertici militari ${ }^{30}$, diventò oltremodo importante definire lo spirito d'appartenenza delle minoranze dei confini orientali. A tale fine, "con i dati numerici del censimento del 1936”, nel 1939 fu condotto “un «censimento segreto» con il quale verrà fatta una stima della presenza degli «allogeni», che mostrerà il fallimento delle pratiche di assimilazione del regime" ${ }^{31}$. I dati raccolti dalle autorità locali e trasmessi all'Istituto centrale di statistica del Regno d'Italia, dimostrano che la struttura nazionale della popolazione della Venezia Giulia nell'anno 1939 non si scostava di molto da quella registrata con il contestato censimento austriaco del lontano $1910 .^{32}$

29 Lavorando negli archivi della Prefettura di Pola, Hrvoje Mezulić ha potuto stabilire ancora nel 1946 che delle ben 26.808 pratiche di cambiamento del cognome (ognuna delle quali concerneva un capo famiglia) solo 16.822 furono spediti a Roma fra il 20 maggio 1929 e 14 ottobre 1937 per la pubblicazione in forma di decreto sulla G.U. Mezulić, Fašizam, krstitelj i palikuća, 66.

30 Idem, 109-110.

31 D'Alessio, “Dall'Impero d'Austria al Regno d'Italia”, 39.

32 Marino Manin, Istra na raskrižju. O povijesti migracija pučanstva Istre, Zagreb 2010, capitolo 6.: "O propasti talijanske politike odnarođivanja u Istri (Tajno popisivanje istarskih Hrvata 1939. godine)”, 103-123. 


\section{La Provincia dell'Istria nei decreti prefettizi pubblicati}

Dopo un inizio caratterizzato da una mutevole geografia amministrativa, la Provincia dell'Istria - al momento del VI censimento generale del 1921 - fu divisa in 5 distretti politici (Capodistria, Parenzo, Pisino, Pola e Lussino) comprensivi di 40 Comuni, che divennero 41 nel 1934 dopo il distacco di Brioni Maggiore dal Comune di Pola.

Le pratiche per l'italianizzazione erano di competenza del prefetto di Pola, ruolo assunto di volta in volta, nel periodo qui trattato, dai seguenti quattro funzionari ${ }^{33}$ :

- Cavalieri dott. Enrico: 16.10.1926 - 01.04.1928 (dapprima prefetto di Vicenza, in seguito prefetto di Udine),

- Leone avv. Leone: 01.04.1928 - 16.05.1931 (dapprima e sino a fine legislatura anche deputato, in seguito prefetto di Foggia),

- Foschi avv. Italo: 16.05.1931 - 10.09.1933 (dapprima prefetto di Macerata, in seguito collocato a disposizione),

- Cimoroni avv. Oreste: 10.09.1933 - 21.08.1939 (ex deputato, nominato in seguito prefetto di Padova).

Tutti e quattro i prefetti citati erano militanti nel P.N.F, furono nominati come "prefetti di $2^{\text {a }}$ classe" ${ }^{44}$ e, eccezion fatta per Enrico Cavalieri, furono tutti "prefetti non di carriera" ${ }^{35}$.

A questi prefetti vanno aggiunti i viceprefetti Roberto Rizzi e Michele Serra nonché un certo Marolla (il nome non è indicato); le loro firme erano precedute dalla formula $p$. Il prefetto. Il ruolo di questi funzionari nel processo d'italianizzazione non fu secondario. Al Dott. Roberto Rizzi, dapprima sottoprefetto a Parenzo poi viceprefetto a Pola fino alla sua partenza per Pescara nel marzo del 1929 dopo la nomina a prefetto di $2^{\text {a }}$ classe $^{36}$, viene attribuita la compilazione dell'elenco dei cognomi da restituire in forma italiana per la Provincia dell'Istria ${ }^{37}$, come imposto dal paragrafo 1 del Decreto ministeriale del 5 agosto 1926. Questo elenco, pubblicato a Pola in un'apposita brochure ${ }^{38}$ per servire da base all'italianizzazione dei cognomi nella provincia dell'Istria, apparse pure a scaglioni fra l'aprile e il

\footnotetext{
33 Mario Missori, Governi, alte cariche dello Stato, alti magistrati e prefetti del Regno d'Italia (in seguito: Governi, alte cariche dello Stato), Roma 1989, 560.

34. Alberto Cifelli, I prefetti del regno nel ventennio fascista, Roma 1999, 67, 75, 122, 123, 151.

35 Idem, 302.

36 Idem, 237, 238; Missori, Governi, alte cariche dello Stato, 552.

37 Milan Nosić, Talijanizacija istarskih prezimena, Rijeka 2010, 9.

38 Josip Bratulić - Petar Šimunović nella prefazione del Prezimena i naselja u Istri, I, 13.
} 
settembre 1928 sul settimanale in lingua croata Istarska rijec ${ }^{39}$. Sulla completezza di quest'ultimo torneremo più oltre.

Stando a una testimonianza da noi raccolta negli anni Ottanta del secolo scorso, nel Montonese circolava pure un opuscolo contenente "l'elenco dei cognomi” che era possibile consultare nel corso della campagna d'italianizzazione del 1928; i cognomi di sostituzione indicati differivano dall'elenco del settimanale.

\subsection{Numero dei decreti pubblicati e di persone coinvolte}

I decreti di riduzione del cognome in forma italiana emessi fra il $1^{\circ}$ marzo 1928 e il 16 marzo 1935 dai quattro prefetti e pubblicati sulla G.U. furono 16.815 (Tabelle 1 e 2) e ufficializzarono i cambiamenti dei cognomi di 70.145 persone - ovvero il $23,82 \%$ dei 294.492 residenti nella provincia secondo l’VIII censimento generale del 21 aprile 1936. Analizzando i numeri di identificazione dei decreti (composti dalla lettera iniziale del cognome vecchio seguita dal numero progressivo delle pratiche avviate), è lecito pensare, considerando la discontinuità della numerazione, che in realtà molti decreti non siano stati pubblicati e che pertanto le persone coinvolte nell'italianizzazione del cognome fossero di fatto molte di più. Una disamina della numerazione delle pubblicazioni mostra infatti che il numero totale dei decreti pubblicati è inferiore al numero massimo indicato dal codice di identificazione di questi decreti, una discrepanza che rivela l'assenza dalla G.U. di almeno 4.500 decreti e che eleverebbe il numero di persone coinvolte a non meno di 90.000. Come si vedrà in seguito, altri elementi contribuiscono a far salire il numero di persone coinvolte dal fenomeno a livelli ancora maggiori.

Nonostante la grande solerzia da parte della prefettura, manifestata anche in termini di persone e mezzi profusi dalla prefettura, la popolazione di questa provincia, tanto nelle campagne quanto nelle città costiere, si rivelò restia a sottoporre una richiesta volontaria di cambiamento del proprio cognome. Come già esposto nella Tabella 2 , solo 1.262 individui $(7,5$ $\%$ del totale) presentarono una richiesta "per la riduzione del cognome in forma italiana”; il rimanente 92,5 \% dei cognomi risulta dunque imposto. Un'opposizione maggiore la troviamo solo nella Provincia di Gorizia dove nessuna richiesta risulta documentata. Una rapida analisi dei decreti emessi

39 Consultabile sul portale Istarske novine online (http://www.ino.com.hr/istarska_rijec.html) oppure nel libro Talijanizacija istarskih prezimena di Nosić. 
su richiesta, rivela che la maggior parte delle domande spontanee fu fatta immediatamente dopo la pubblicazione dell'elenco; che di quelle provenienti dai centri urbani un numero cospicuo riguarda le persone singole; e che infine quelle meno numerose dalle campagne riguardano le famiglie con figli in età adulta. Nel periodo successivo buona parte delle domande provennero da residenti nelle campagne e più precisamente da genitori, fratelli o sorelle dei primi richiedenti.

\subsection{Ritmi d'emissione e di pubblicazione dei decreti}

In base alla data d'emissione (indicata in tutti i decreti pubblicati) e al nome del prefetto o del viceprefetto firmatario (figurante solo sui decreti pubblicati in forma integrale, ossia il 73,27 \%) è possibile seguire i ritmi di emissione e di pubblicazione dei decreti nei diversi periodi prefettizi.

La Tabella 4 raggruppa i decreti secondo il periodo prefettizio e i firmatari per evidenziare il ruolo svolto dai viceprefetti. Oltre il $90 \%$ dei decreti furono emessi in poco più di cinque anni, ovvero nel periodo dei prefetti Leone Leone e Italo Foschi, i maggiori firmatari.

Come già accennato, lo spazio richiesto da ciascun decreto sulla G.U. era tale che le pubblicazioni non riuscivano a seguire i ritmi d'emissione. Il problema d'accumulo dei decreti non pubblicati toccò tutte le province, ma in modo particolare quella dell'Istria. La Tabella 5 mette a confronto, anno

\begin{tabular}{|c|c|c|c|c|c|c|c|c|c|c|}
\hline $\begin{array}{l}\text { Periodo } \\
\text { firme } \\
\text { Periodo } \\
\text { prefettizio }\end{array}$ & $\begin{array}{c}\text { Enrico } \\
\text { Cavalieri } \\
01.03 .1928 \\
10.04 .1928\end{array}$ & \begin{tabular}{|c|} 
Leone Leone \\
23.04 .1928 \\
07.07 .1931
\end{tabular} & $\begin{array}{c}\text { Italo } \\
\text { Foschi } \\
12.05 .1931 \\
18.05 .1933\end{array}$ & $\begin{array}{c}\text { Roberto } \\
\text { Rizzi } \\
06.04 .1928 \\
31.12 .1929\end{array}$ & $\begin{array}{c}\text { Michele } \\
\text { Serra } \\
\text { 09.05.1930 } \\
21.05 .1932\end{array}$ & $\begin{array}{c}\text { Marolla } \\
17.05 .1932 \\
19.05 .1932\end{array}$ & $\begin{array}{c}\text { Non indicato } \\
04.02 .1932 \\
16.03 .1935\end{array}$ & $\begin{array}{c}\text { Totale } \\
\text { periodo } \\
\text { prefettizio }\end{array}$ & $\begin{array}{c}\% \\
\text { x periodo } \\
\text { prefettizio }\end{array}$ & $\begin{array}{l}\text { Persone } \\
\text { coinvolte }\end{array}$ \\
\hline $\begin{array}{l}\text { Enrico } \\
\text { Cavalieri } \\
16.10 .1926 \\
30.03 .1928\end{array}$ & 21 & & & & & & & 21 & 0,12 & 75 \\
\hline $\begin{array}{l}\text { Leone Leone } \\
01.04 .1928 \\
15.05 .1931\end{array}$ & 4 & 7.475 & 4 & 1.383 & 611 & & & 9.477 & 56,36 & 38.427 \\
\hline $\begin{array}{l}\text { Italo Foschi } \\
16.05 .1931 \\
10.09 .1933\end{array}$ & & 3 & 2.287 & & 497 & 35 & 3.167 & 5.989 & 35,62 & 25.480 \\
\hline $\begin{array}{l}\text { Oreste } \\
\text { Cimoroni } \\
10.09 .1933 \\
21.08 .1939\end{array}$ & & & & & & & 1.328 & 1.328 & 7,90 & 6.163 \\
\hline $\begin{array}{l}\text { Totale } \\
\text { decreti } \\
\text { x firmatari }\end{array}$ & 25 & 7.478 & 2.291 & 1.383 & 1.108 & 35 & 4.495 & 16.815 & & \\
\hline $\begin{array}{l}\text { \% x periodo } \\
\text { prefettizio }\end{array}$ & 0,15 & 44,47 & 13,62 & 8,22 & 6,59 & 0,21 & 26,73 & & 100,00 & \\
\hline $\begin{array}{l}\text { Persone } \\
\text { coinvolte }\end{array}$ & 95 & 30.813 & 9.427 & 5.581 & 4.073 & 126 & 20.030 & & & 70.145 \\
\hline
\end{tabular}


per anno, i decreti emessi dalla Prefettura di Pola col totale di quelli effettivamente pubblicati. A fronte degli 11.471 decreti emessi nei primi quattro anni (68,22 \% del totale), soltanto 5.069 furono pubblicati in quel periodo, mentre oltre la metà (6.402 decreti) restò in attesa di pubblicazione. Il numero degli atti cui toccò lo stesso destino si sarebbe mantenuto altissimo nei quattro anni seguenti. Si cercò di porvi rimedio nel 1936 con la pubblicazione di 3.791 decreti (22,55 \% del totale) in una sbrigativa forma tabellare. Di questi, 836 erano stati emessi nel 1932. Per nessun'altra provincia si registrano simili ritardi.

Va notato che il pareggio raggiunto nel 1936 riguardò soltanto i decreti da noi esaminati mentre, come detto sopra, altri decreti, benché emessi, non furono mai pubblicati in violazione pertanto dell'art. 1 del R.D.L. 17.

\begin{tabular}{|c|c|c|c|c|c|c|c|}
\hline Anno & $\begin{array}{l}\text { Decreti } \\
\text { secondo data } \\
\text { emissione }\end{array}$ & $\%$ & $\begin{array}{l}\text { Decreti } \\
\text { secondo data } \\
\text { pubblicazione }\end{array}$ & $\%$ & $\begin{array}{l}\text { Cumulativo } \\
\text { emessi }\end{array}$ & $\begin{array}{l}\text { Cumulativo } \\
\text { pubblicati }\end{array}$ & $\begin{array}{l}\text { Decreti in } \\
\text { attesa di } \\
\text { pubblicazione }\end{array}$ \\
\hline 1928 & 1.800 & 10,71 & 1.277 & 7,59 & 1.800 & 1.277 & -523 \\
\hline 1929 & 1.854 & 11,03 & 939 & 5,58 & 3.654 & 2.216 & -1.438 \\
\hline 1930 & 3.330 & 19,80 & 914 & 5,44 & 6.984 & 3.130 & -3.854 \\
\hline 1931 & 4.487 & 26,68 & 1.939 & 11,53 & 11.471 & 5.069 & -6.402 \\
\hline 1932 & 2.388 & 14,20 & 2.749 & 16,35 & 13.859 & 7.818 & -6.041 \\
\hline 1933 & 1.858 & 11,05 & 2.586 & 15,38 & 15.717 & 10.404 & -5.313 \\
\hline 1934 & 968 & 5,76 & 1.161 & 6,90 & 16.685 & 11.565 & -5.120 \\
\hline 1935 & 130 & 0,77 & 1.459 & 8,68 & 16.815 & 13.024 & -3.791 \\
\hline 1936 & 0 & 0,00 & 3.791 & 22,55 & 16.815 & 16.815 & 0 \\
\hline Totale & 16.815 & 100,00 & 16.815 & 100,00 & & & \\
\hline
\end{tabular}

Tabella 5. Confronto tra i decreti emessi e quelli pubblicati per la Prefettura di Pola

\subsection{Distribuzione geografica delle persone coinvolte secondo it comune di residenza}

Informazioni interessanti emergono dall'analisi della distribuzione geografica delle persone coinvolte dal fenomeno di italianizzazione. Il comune di residenza è ricavabile per il $70 \%$ degli individui o perché esplicitato o perché deducibile dal luogo di nascita dei figli. Per il restante $30 \%$ è stato adottato, come comune di residenza, il comune di nascita del titolare. I dati così ottenuti sono messi a confronto col numero di abitanti secondo l'VIII censimento generale del 21 aprile 1936 e riportati nella Tabella 6. 


\begin{tabular}{|c|c|c|c|c|c|c|c|c|c|c|}
\hline \multirow[b]{2}{*}{$\begin{array}{l}\text { Distretto } \\
\text { politico }\end{array}$} & \multirow[b]{2}{*}{ Comune } & \multicolumn{5}{|c|}{ Residenza persone coinvolte } & \multicolumn{2}{|c|}{ Abitanti 1936} & \multicolumn{2}{|c|}{$\%$ coinvolti } \\
\hline & & 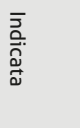 & 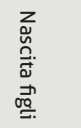 & 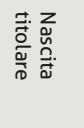 & 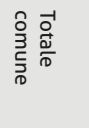 & 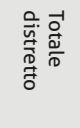 & $\begin{array}{l}0 \\
\stackrel{0}{3} \\
\frac{1}{5}\end{array}$ & 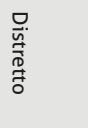 & 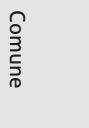 & 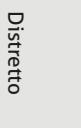 \\
\hline \multirow{10}{*}{ CAPODISTRIA } & Capodistria & 1.477 & 888 & 1.131 & 3.496 & & 11.995 & & 29,14 & \\
\hline & Erpelle Cosina & 216 & 61 & 541 & 818 & & 2.399 & & 34,10 & \\
\hline & Isola d’Istria & 19 & 5 & 92 & 116 & & 9.771 & & 1,19 & \\
\hline & Lanischie & 13 & 6 & 122 & 141 & & 3.480 & & 4,05 & \\
\hline & Maresego & 9 & 6 & 47 & 62 & & 3.518 & & 1,76 & \\
\hline & Monte di Capod. & 10 & 0 & 0 & 10 & & 4.820 & & 0,21 & \\
\hline & Pinguente & 90 & 32 & 89 & 211 & & 10.222 & & 2,06 & \\
\hline & Pirano & 618 & 6 & 311 & 935 & & 15.117 & & 6,19 & \\
\hline & Rozzo & 121 & 110 & 125 & 356 & & 2.743 & & 12,98 & \\
\hline & Villa Decani & 142 & 3.404 & 1.724 & 5.270 & 11.415 & 6.808 & 70.873 & 77,41 & 16,10 \\
\hline \multirow{5}{*}{ LUSSINO } & Cherso & 964 & 1.291 & 260 & 2.515 & & 7.570 & & 33,22 & \\
\hline & Lussingrande & 676 & 147 & 58 & 881 & & 1.992 & & 44,23 & \\
\hline & Lussinpiccolo & 1.696 & 34 & 510 & 2.240 & & 6.856 & & 32,67 & \\
\hline & Neresine & 1.643 & 36 & 57 & 1.736 & & 1.904 & & 91,18 & \\
\hline & Ossero & 739 & 0 & 36 & 775 & 8.147 & 1.047 & 19.369 & 74,02 & 42,06 \\
\hline \multirow{11}{*}{ PARENZO } & Buie d'Istria & 2.558 & 34 & 107 & 2.699 & & 7.293 & & 37,01 & \\
\hline & Cittanova d'Istria & 54 & 0 & 26 & 80 & & 2.517 & & 3,18 & \\
\hline & Grisignana & 669 & 0 & 995 & 1.664 & & 3.977 & & 41,84 & \\
\hline & Montona & 1.643 & 16 & 766 & 2.425 & & 6.692 & & 36,24 & \\
\hline & Orsera & 124 & 22 & 60 & 206 & & 5.565 & & 3,70 & \\
\hline & Parenzo & 176 & 44 & 1.992 & 2.212 & & 12.036 & & 18,38 & \\
\hline & Portole & 401 & 520 & 162 & 1.083 & & 5.709 & & 18,97 & \\
\hline & Umago & 18 & 8 & 408 & 434 & & 7.112 & & 6,10 & \\
\hline & Verteneglio & 37 & 3 & 3 & 43 & & 3.242 & & 1,33 & \\
\hline & Visignano d'Istria & 111 & 0 & 102 & 213 & & 5.071 & & 4,20 & \\
\hline & Visinada & 148 & 9 & 39 & 196 & 11.255 & 4.998 & 64.212 & 3,92 & 17,53 \\
\hline \multirow{7}{*}{ PISINO } & Albona & 83 & 24 & 232 & 339 & & 16.973 & & 2,0 & \\
\hline & Antignana & 95 & 2 & 260 & 357 & & 4.268 & & 8,36 & \\
\hline & Bogliuno & 362 & 10 & 30 & 402 & & 3.843 & & 10,46 & \\
\hline & Fianona & 169 & 11 & 487 & 667 & & 4.687 & & 14,23 & \\
\hline & Gimino & 3.928 & 530 & 172 & 4.630 & & 5.891 & & 78,59 & \\
\hline & Pisino & 1.768 & 762 & 4.185 & 6.715 & & 19.094 & & 35,17 & \\
\hline & Valdarsa & 0 & 3 & 23 & 26 & 13.136 & 2.158 & 56.914 & 1,20 & 23,08 \\
\hline \multirow{8}{*}{ POLA } & Barbana d'Istria & 20 & 6 & 2.199 & 2.225 & & 5.366 & & 41,46 & \\
\hline & Brioni Maggiore & 58 & 0 & 0 & 58 & & 310 & & 18,71 & \\
\hline & Canfanaro & 921 & 182 & 1.010 & 2.113 & & 3.894 & & 54,26 & \\
\hline & Dignano d'Istria & 2.024 & 33 & 844 & 2.901 & & 11.265 & & 25,75 & \\
\hline & Pola & 11.688 & 1.073 & 1.096 & 13.857 & & 46.259 & & 29,96 & \\
\hline & Rovigno d'Istria & 793 & 589 & 134 & 1.516 & & 10.028 & & 15,12 & \\
\hline & Sanvincenti & 2.051 & 5 & 66 & 2.122 & & 3.445 & & 61,60 & \\
\hline & Valle d’Istria & 7 & 174 & 536 & 717 & 25.509 & 2.557 & 83.124 & 28,04 & 30,69 \\
\hline FUORI PROV. & Fuori provincia & 192 & 0 & 0 & 192 & 192 & 0 & & 0 & 0 \\
\hline NON DEFINITO & Nati fuori prov. & 0 & 0 & 491 & 491 & 491 & 0 & & 0 & 0 \\
\hline PROVINCIA & & 38.531 & 10.086 & 21.528 & 70.145 & 70.145 & 294.492 & 294.492 & 23,82 & 23,82 \\
\hline
\end{tabular}

Tabella 6. Distribuzione geografica secondo il comune di residenza delle persone coinvolte 


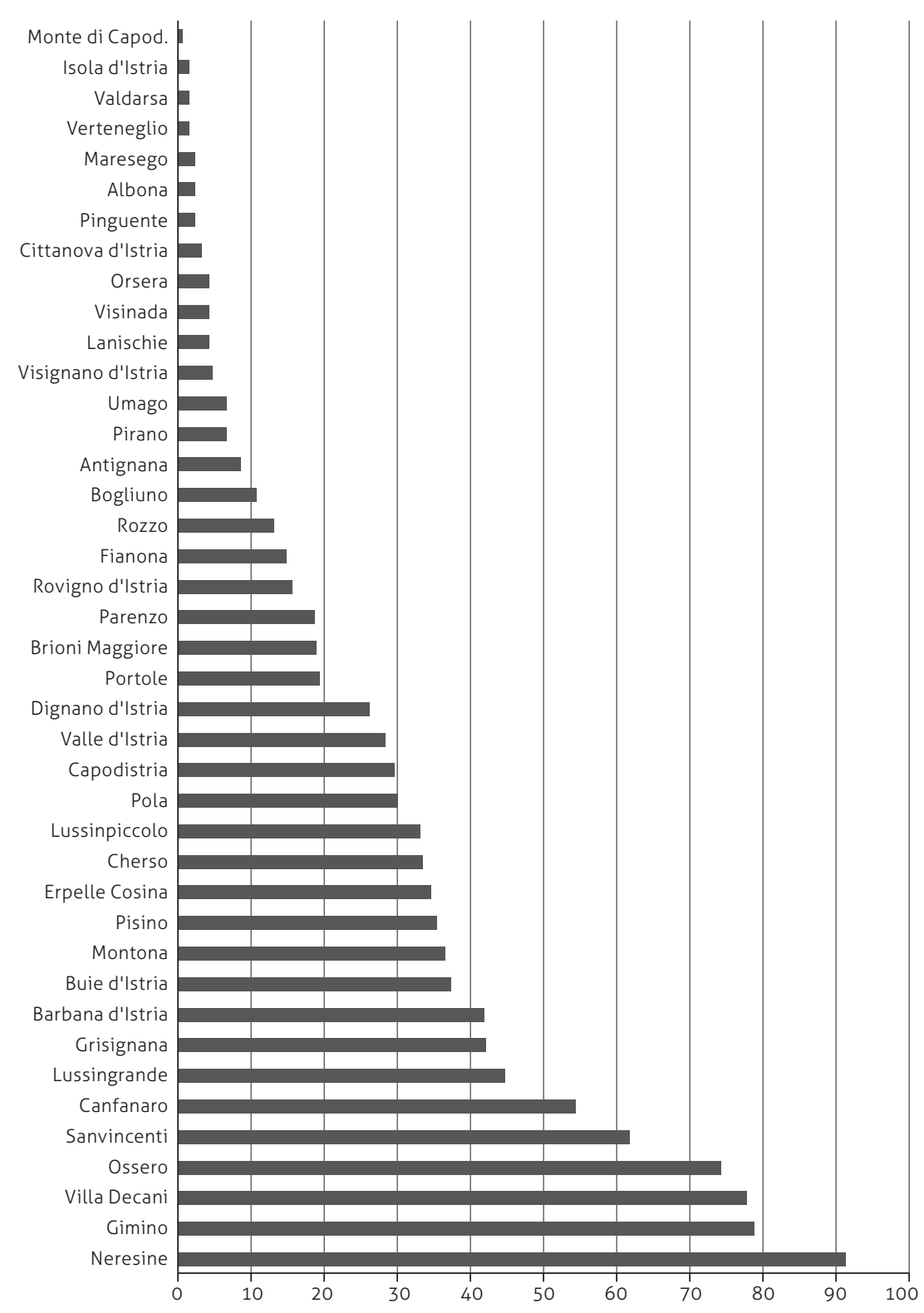

Grafico 1. Numero di persone coinvolte per 100 abitanti

Si evidenzia così che il distretto maggiormente colpito fu quello di Lussino dove l'italianizzazione forzata toccò il 42,06 \% della popolazione residente. Considerevoli sono altresì le percentuali registrate per gli altri distretti: Pola (30,69 \%), Pisino (23,08 \%), Parenzo (17,53\%) e Capodistria (16,10\%). (Mappa 1)

In ogni distretto i singoli comuni denotano percentuali fortemente oscillanti rispetto alla media del distretto. Il Grafico 1 riporta le percentuali 
degli individui coinvolti nei 41 comuni della provincia dell'Istria. Di questi i cinque più colpiti furono Neresine (91,18 \% degli abitanti), Gimino (78,59 \%), Villa Decani (77,41 \%), Ossero (74,02 \%), Sanvincenti (61,60 \%). Il comune meno interessato dal fenomeno fu Monte di Capodistria (0,21\%).

Va sottolineato che la bassa percentuale di persone coinvolte nei comuni periferici, abitati prevalentemente da sloveni e/o croati (Monte di Capodistria, Maresego, Pinguente, Lanischie e altri), può fornire un’immagine ingannevole sia della distribuzione etnica in questi comuni sia dello sforzo fascista nel portare avanti il loro disegno politico: dai dati censiti nel 1945 risultano infatti italianizzati i cognomi di moltissimi altri individui.

A titolo d'esempio sono confrontati i decreti pubblicati riguardanti il comune di Lanischie (Lanišće) - inteso secondo la geografia amministrativa del tempo - con la situazione onomastica ivi registrata dalla commissione preposta al censimento del $1^{\circ}$ ottobre $1945^{40}$.

Al momento dell'VIII censimento generale del 21 aprile 1936 questo comune contava 3.480 abitanti residenti distribuiti nelle frazioni di Lanischie (Lanišće), Bregozza (Brgudac), Ràcia (Račja Vas), Praporchie (Prapoće), Podgacchie (Podgaće), Danne (Dane), Silun Mont’Aquila (Slum), Olmeto (Brest), Trestenico (Trstenik), Raspo (Rašpor) e Clenosciacco (Klenovšćak). Al $1^{\circ}$ ottobre 1945 queste stesse frazioni - tornate a far parte, come nel periodo di dominazione asburgica, del comune di Pinguente (Buzet) - contavano 3.299 abitanti dei quali 3.286 si dichiaravano croati, 9 italiani e 4 sloveni. Seguendo i criteri adottati per la differenziazione dei $\operatorname{cognomi}{ }^{41}$, gli abitanti di questo comune furono censiti riportandone sia il cognome originale sia quello impostogli con l'italianizzazione. Dei 699 nuclei famigliari censiti (individui stimati 2.574) 546 portavano il cognome italianizzato - in netto contrasto con i 34 decreti pubblicati sulla G.U. (141 individui).

I cognomi più ricorrenti dei 74 presenti al $1^{\circ}$ ottobre 1945 nelle frazioni dell'ex comune di Lanischie sono analizzati nella Tabella 7. Un loro raffronto coi decreti pubblicati evidenzia non solo l'arbitraria applicazione della legge, ma altresì un iter al di fuori della legge stessa dal momento che non ci si premurava di documentare ufficialmente i cambiamenti. Secondo Parovel già nei primi anni Venti in Istria si "procedette a veri e propri atti d'imperio, ordinando agli uffici parrocchiali e scolastici la modifica d'iscri-

40 Cadastre national de l'Istrie: d'après le Recensement du $1^{\text {er }}$ Octobre 1945, a cura di Josip Roglić et al., Sušak 1946,474 e 475 .

41 Idem, VII. 
zione anagrafica dei cognomi non italiani, secondo liste preordinate" ${ }^{\prime \prime 2}$. Questo percorso parallelo deve essere tenuto presente se si vuole raggiungere una stima veridica della popolazione coinvolta nel fenomeno dell'italianizzazione. È pertanto legittimo ipotizzare che nella Provincia dell'Istria gli individui toccati non solo andarono oltre i 70.145 abitanti residenti come desunto dai decreti pubblicati, ma sicuramente superarono la cifra di 90.000 unità citata in precedenza.

\begin{tabular}{|l|c|c|c|c|c|c|}
\hline $\begin{array}{c}\text { Residenti nelle frazioni dell'ex comune di } \\
\text { Lanischie censiti al 1.10.1945 }\end{array}$ & \multicolumn{4}{|c|}{ Decreti pubblicati sulla G.U. } \\
\hline Cognome indicato & Famiglie & $\begin{array}{c}\text { Persone } \\
\text { stimate }\end{array}$ & $\begin{array}{c}\text { Decreti comune } \\
\text { Lanischie }\end{array}$ & $\begin{array}{c}\text { Decreti altri } \\
\text { comuni }\end{array}$ & $\begin{array}{c}\text { Persone } \\
\text { coinvolte } \\
\text { Lanischie }\end{array}$ & $\begin{array}{c}\text { Persone coinvolte } \\
\text { altri comuni }\end{array}$ \\
\hline Božić (Bossi) & 95 & 448 & 6 & 88 & 29 & 344 \\
Poropat (Poretti) & 79 & 372 & 0 & 69 & 0 & 284 \\
Šverko (Sferco) & 68 & 321 & 2 & 7 & 10 & 32 \\
Grbac (Gherbassi) & 50 & 236 & 0 & 24 & 0 & 80 \\
Črnac (Cerna) & 34 & 160 & 2 & 27 & 5 & 131 \\
Brajković (Braico) & 29 & 137 & 2 & 51 & 9 & 299 \\
Ivančić (Giovannini) & 27 & 127 & 1 & 91 & 4 & 402 \\
\hline Totale & 382 & 1.801 & 13 & 357 & 57 & 1.572 \\
\hline
\end{tabular}

Tabella 7. Cognomi più ricorrenti al $1^{\circ}$ ottobre 1945 nelle frazioni dell'ex comune di Lanischie, a confronto con i decreti pubblicati sulla G.U.

\subsection{Categorie professionali dei titolari}

La professione del titolare del decreto è indicata solo su 1.070 decreti pubblicati (6,36 \%), per un totale di 4.161 individui. Curiosamente questa indicazione viene riportata quasi esclusivamente sui decreti emessi su richiesta dell'interessato, ossia in 1.021 casi $(95,4 \%)$ riportati nel Grafico 2.

La categoria più numerosa è quella degli agricoltori con 278 richieste delle quali ben 112 nel solo distretto giudiziario di Montona. Per meglio capire il fenomeno e memori della testimonianza citata più sopra, abbiamo consultato due settimanali dell'epoca che, redatti in lingua croata, rappresentavano una possibile fonte d'informazione per la popolazione non di lingua italiana.

Il Pučki prijatelj, un settimanale fondato nel lontano 1899, vedendosi sbiancare molti spazi dalla censura fra il settembre 1919 e il marzo 1920, assunse toni sempre più pacati nelle questioni politiche, tanto che nel gen-

42 Parovel, Izbrisani identitet, 25; pure Bratulić - Šimunović nella prefazione del Prezimena i naselja u Istri, I, 


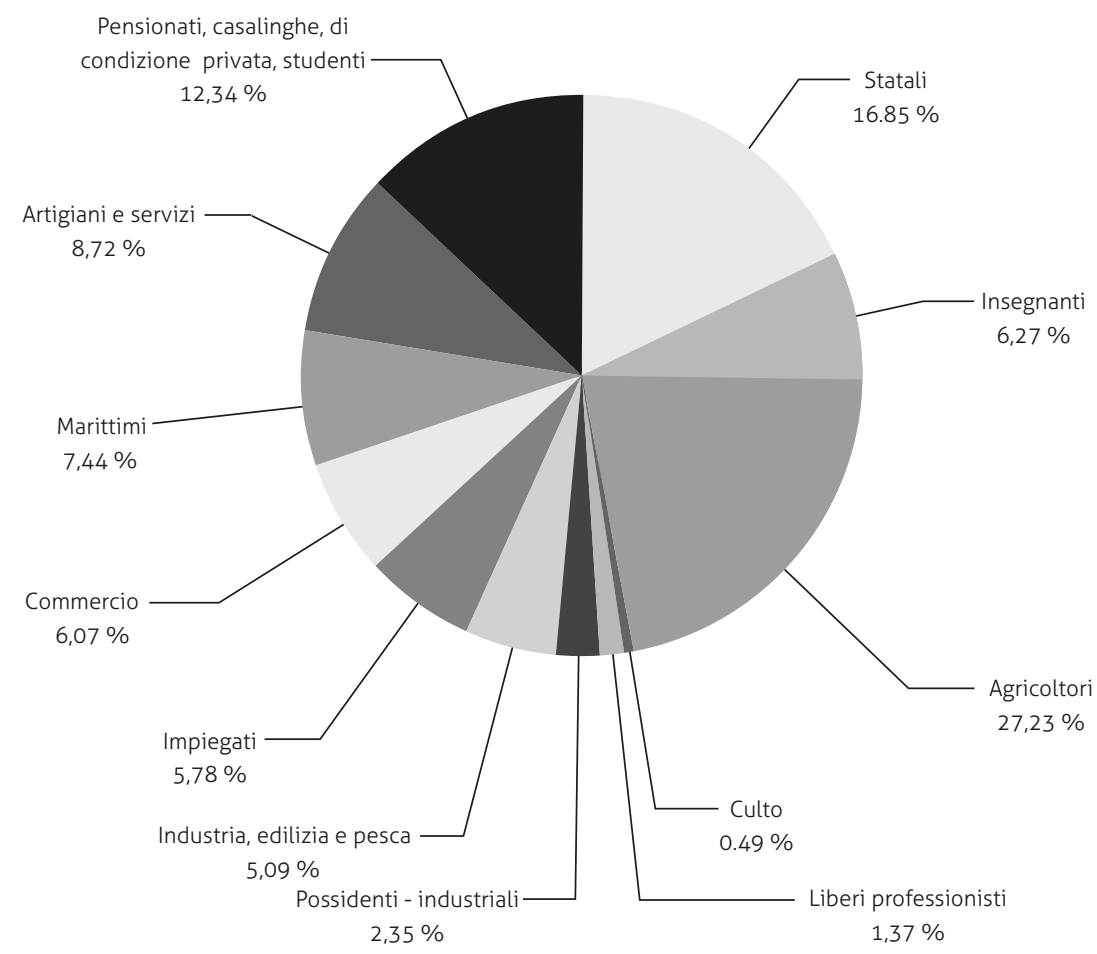

Grafico 2. I titolari dei decreti su domanda secondo le profiessioni

naio 1926, riferì del R.D.L. N. 17 concernente i cognomi della Provincia di Trento con una notizia telegrafica in terza pagina ${ }^{43}$; in seguito, nell'ottobre dello stesso anno, con una sola frase informava della sollecitazione de Il Piccolo di Trieste affinché il detto R.D.L. venisse quanto prima esteso alle province della Venezia Giulia ${ }^{44}$. All'argomento fu dedicato ampio spazio due settimane più tardi, quando il settimanale riferì delle modalità delle proposte de Il Piccolo, ma dichiarò che "per ovvi motivi non si descrivono i sentimenti” della minoranza slava nel caso tali proposte fossero accettate ${ }^{45}$. Seguì un lungo silenzio interrotto solo nell'aprile 1927 da una stringata notizia sulla proposta ufficiale d'estensione del R.D.L. 17 alle altre province $^{46}$ e nell'ottobre 1928 da una altrettanto breve notizia sull'avvio, nella Provincia di Bolzano, dell'imposizione d'ufficio dei cognomi italiani in sostituzione di quelli tedeschi in base ad una lista finora pubblicata per le iniziali

43 “Mijenjanje obiteljskog prezimena”, Pučki prijatelj, n. 5 del 28 gennaio 1926, 3, http://www.nsk.hr/starehrvatske-novine (visto maggio 2017).

44. "Popravak prezimena", idem, n. 41 del 7 ottobre 1926, 1.

45 "Naša prezimena”, idem, n. 43 del 21 ottobre 1926, 2.

46 "Promjena prezimena", idem, n. 14 del 7 aprile 1927, 1. 
"A" e "B" $"$ " Nessun accenno tantomeno commento al fatto che per l'Istria l'elenco fosse già finito e pubblicato. Il settimanale sarà soppresso dopo il numero del 10 gennaio 1929.

L'Istarska riječ, settimanale in lingua croata fondato nel gennaio del 1923, fu pure soppresso dopo il numero del 10 gennaio 1929. Il periodico toccò l'argomento dei cognomi due volte: nell'aprile 1927 (dapprima con una breve notizia sul R.D. $494^{48}$, poi con qualche informazione in più nel numero seguente $^{49}$ ) dopodiché resta silente sul tema fino al 12 aprile 1928 quando, iniziata la pubblicazione dell'elenco compilato per la Provincia dell'Istria, spiega i meccanismi d'applicazione della legge (informando pure che fra tutte le prefetture tenute a compilare l'elenco "la commissione formatasi presso la Prefettura di Pola risulta essere la prima ad aver compiuto il suo lavoro”), ma senza commenti, limitandosi a una breve dichiarazione: "Troviamo che la lista sia sotto diversi punti di vista interessante, anzi molto interessante". ${ }^{50}$

Frequenti e dettagliate, per contro, apparivano su entrambi i settimanali le notizie sulle difficili condizioni di sopravvivenza degli agricoltori del nord istriano (ma non solo): quelli che potevano emigravano in Argentina mentre per chi restava l'unica speranza erano saltuarie occasioni d'occupazione nei lavori pubblici (bonifica della Valle di Quieto, costruzione dell'acquedotto di Pinguente, diversi lavori stradali) e per accedervi un cognome italiano era d'obbligo.

Fra i 49 decreti con la professione indicata e imposti d'ufficio, emerge un altro particolare interessante: ben 40 di questi riguardavano i sacerdoti. Questi erano nativi principalmente della provincia stessa (23); gli altri provenivano dalle province vicine (Trieste 7, Gorizia 1, Carnaro 1) oppure fuori dai confini nazionali (Regno di SHS 7 e Cechia 1). Luogo di residenza: Pisino 9, Capodistria 5, Lussino 5, Albona 3, Dignano 3, Pola 3, Montona 2, Parenzo 2, Pinguente 2, Pirano 1, Rovigno 1, Buie 1, Roma 1, senza indicazione 2 . L'analisi delle date d'emissione dei decreti a loro riferiti evidenzia le divergenze sorte fra il clero cattolico e il regime "sull'opera

\footnotetext{
47 "Promjena prezimena u Poadižju”, idem, n. 40 del 4 ottobre 1928, 2.

48 "Prezimena", Istarska riječ, n. 14 del 7 aprile 1927, 2.

49 “Novi zakon o prezimenima”, idem, n. 15 del 14 aprile 1927, 1.

50 "O promijeni prezimena", idem, n. 15 del 12 aprile 1928, 1 e 2.
} 
di snazionalizzazione" ${ }^{51}$. Inaspritesi dopo la firma dei Patti lateranensi tra l'Italia e la Santa Sede nel febbraio 1929, queste divergenze durarono fino all'anno 1931 quando le mire del regime "ad avere nella regione gerarchie ecclesiastiche non ostili alla politica di snazionalizzazione” erano pressoché raggiunte $^{52}$. Negli anni 1929 e 1930 risultano imposti agli ecclesiastici solo 4 cambiamenti di cognome. L’anno 1931 iniziò con un decreto nel mese di febbraio e uno in marzo, ma vide una drastica svolta nel mese d'aprile quando, subito dopo la Domenica di Pasqua, vennero emessi 27 decreti in soli due giorni (14 e 16 aprile) seguiti, tre settimane più tardi, dai decreti riguardanti i 3 sacerdoti residenti a Pola. L'ultimo decreto di un cambio d'ufficio del cognome di un ecclesiastico porta la data 8 febbraio 1933 e riguarda un sacerdote nativo di Trieste, ordinato sacerdote nel maggio dell'anno 1932, e assegnato alla parrocchia di Vetta presso Pinguente. Le pubblicazioni dei decreti concernenti gli ecclesiastici risultano concentrate fra l'11 e il 19 ottobre 1933.

\subsection{Il comune di Pola e la città di Pola}

Dopo aver considerato le dinamiche dell'italianizzazione a livello della provincia dell'Istria, dedichiamo una breve sezione a un'analisi più approfondita per il comune di Pola. Il capoluogo istriano, secondo il censimento del 21 aprile 1936, comprendeva oltre alla città di Pola anche le frazioni di Sichici, Giadreschi, Valdebecco, Vincural, Stignano di Pola, Fasana d'Istria, Peroi, Gallesano, Lavarigo, Monticchio, Altura, Cavrano, Sissano, Lisignano, Medolino, Pomer, Bagnole e Promontore. Liitalianizzazione forzata risultante dai decreti pubblicati viene riportata nella Tabella 8 mentre il Grafico 3 illustra il rapporto percentuale tra gli abitanti coinvolti e il totale di quelli residenti.

\begin{tabular}{|l|r|r|r|}
\hline Frazioni Comune di Pola & $\begin{array}{l}\text { Popolazione residente al } \\
21 \text { aprile 1936 }\end{array}$ & $\begin{array}{l}\text { Persone coinvolte } \\
\text { secondo G.U. }\end{array}$ & $\begin{array}{l}\text { \% persone coinvolte su } \\
\text { residenti }\end{array}$ \\
\hline Pola & 34.090 & 9.500 & 27,87 \\
Sichici & 318 & 116 & 36,48 \\
Giadreschi & 360 & 151 & 41,94 \\
Valdebecco & 174 & 151 & 86,78 \\
Vincural & 451 & 6 & 1,33 \\
Stignano di Pola & 983 & 286 & 29,09 \\
Fasana d'Istria & 1.090 & 314 & 28,81 \\
\hline
\end{tabular}

51 Apih, Italia, fascismo e antifascismo, 285-293.

52 Ibidem. 


\begin{tabular}{|l|r|r|r|}
\hline Peroi & 380 & 143 & 37,63 \\
Gallesano & 1.693 & 176 & 10,40 \\
Lavarigo & 366 & 102 & 27,87 \\
Monticchio & 382 & 82 & 21,47 \\
Altura & 1.261 & 616 & 48,85 \\
Cavrano & 298 & 74 & 24,83 \\
Sissano & 995 & 298 & 29,95 \\
Lisignano & 911 & 485 & 53,24 \\
Medolino & 1.157 & 724 & 62,58 \\
Pomer & 389 & 231 & 59,38 \\
Bagnole & 456 & 98 & 21.49 \\
Promontore & 505 & 304 & 60.20 \\
\hline Comune di Pola & 46.259 & 13.857 & 29,96 \\
\hline
\end{tabular}

Tabella 8. Persone residenti nelle frazioni del Comune di Pola coinvolte nell'italianizzazione forzata

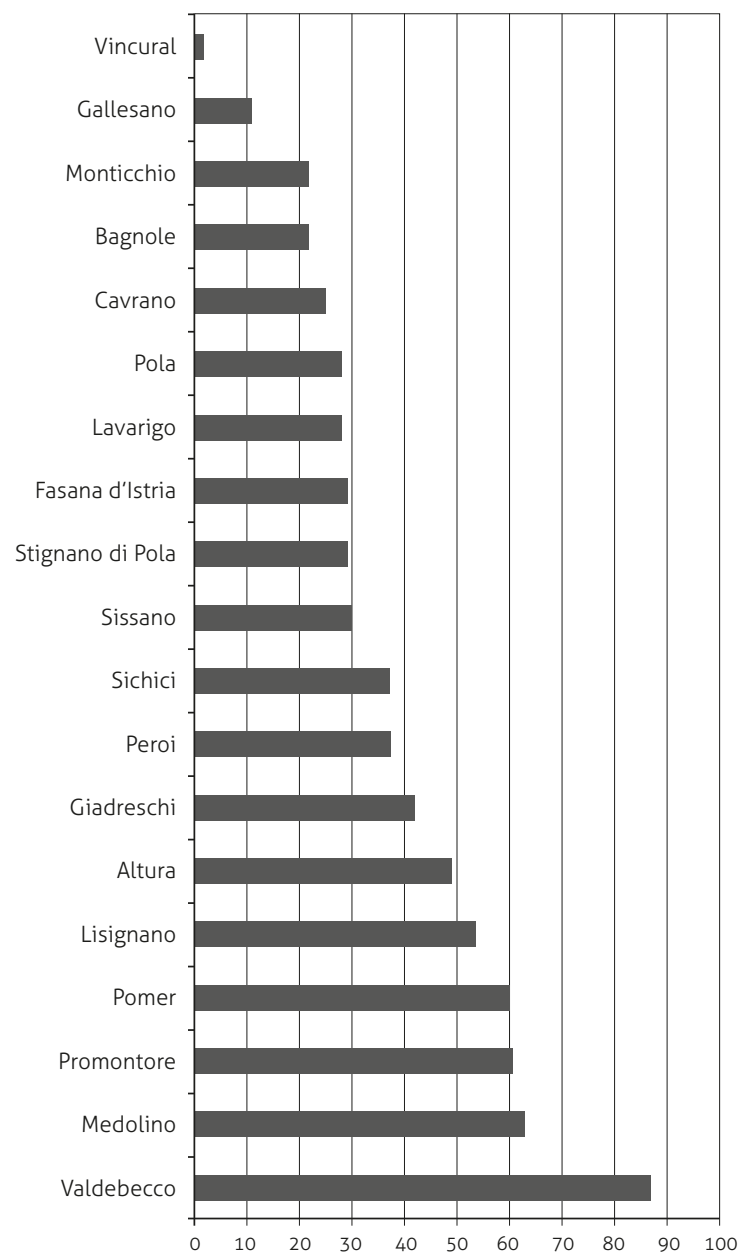

Grafico 3. Coinvolti su 100 abitanti residenti al 21.04.1936 nel Comune di Pola 
Ispezionando la tabella e il grafico, si vede che il fenomeno riguardò sia le frazioni sia Pola Città. Giova, a proposito, considerare l'evoluzione demografica di quest'ultima che nel 1859 fu scelta come principale porto militare dell'Impero Asburgico. La nuova posizione strategica le consentì di crvvescere rapidamente e da modesto borgo che nell'anno 1850 contava solo 1.109 anime si trasformò in una realtà plurilinguistica di 58.562 abitanti i quali nel censimento del 1910 si dichiaravano di lingua tedesca (9.064), italiana (26.816), slovena (3.293), serbocroata (9.511), o parlanti altre lingue (1.634) oppure stranieri $(8.244)^{53}$. Questa crescita demografica si arrestò e la tendenza si invertì dopo l'annessione al Regno d'Italia quando Pola Città visse il suo primo grande esodo. Nel censimento del $1^{\circ}$ dicembre 1921 si registrò una vera e propria emorragia, un calo di 21.495 abitanti $(36,7 \%)$ rispetto al 1910. Le "fragili componenti di multiculturalità e cosmopolitismo”, sbocciate in sette decenni di spettacolare sviluppo, appassivano "assieme alla grande monarchia dei dodici popoli” ${ }^{4}$. In forma minore, ma pur sempre drammatica, ne risentì tutto il distretto dove in media si ebbe una contrazione del 22,2\%.

Il fenomeno rallentò, in seguito la città si ripopolò cosicché nel 1936 contava 34.090 abitanti. Di questi, ben 9.500 (28\%) risultano nei decreti pubblicati sulla G.U. con poche postulazioni spontanee (963) e tante imposizioni (8.537).

Interessante è inoltre la provenienza degli abitanti toccati dall'italianizzazione forzata. Essa è riportata nel Grafico 4: la maggioranza era nata nel distretto di Pola (48\%) o nel vicino distretto di Pisino (30 \%).

Dai 2.470 decreti (corrispondenti a 7.889 persone residenti a Pola Città) che specificano anche l'indirizzo, è possibile dedurre la distribuzione per rione dei cognomi soggetti all’italianizzazione forzata (Grafico 5) a dimostrazione della loro presenza in tutta la città.

53 Spezialortsrepertorium der Österreichischen Länder. Bearbeitet auf Grund der Ergebnisse der Volkszählung vom 31. Dezember 1910. Herausgegeben von der K. K. Statistischen Zentralkommission, VII, Österreichisch-Illyrische Kïstenland, Wien 1918, 54 .

54 Igor Duda, "Elementi kozmopolitizma u Puli između 1850. i 1918. godine”, Radovi Zavoda za hrvatsku povijest, 32-33, 1999-2000, 114. 


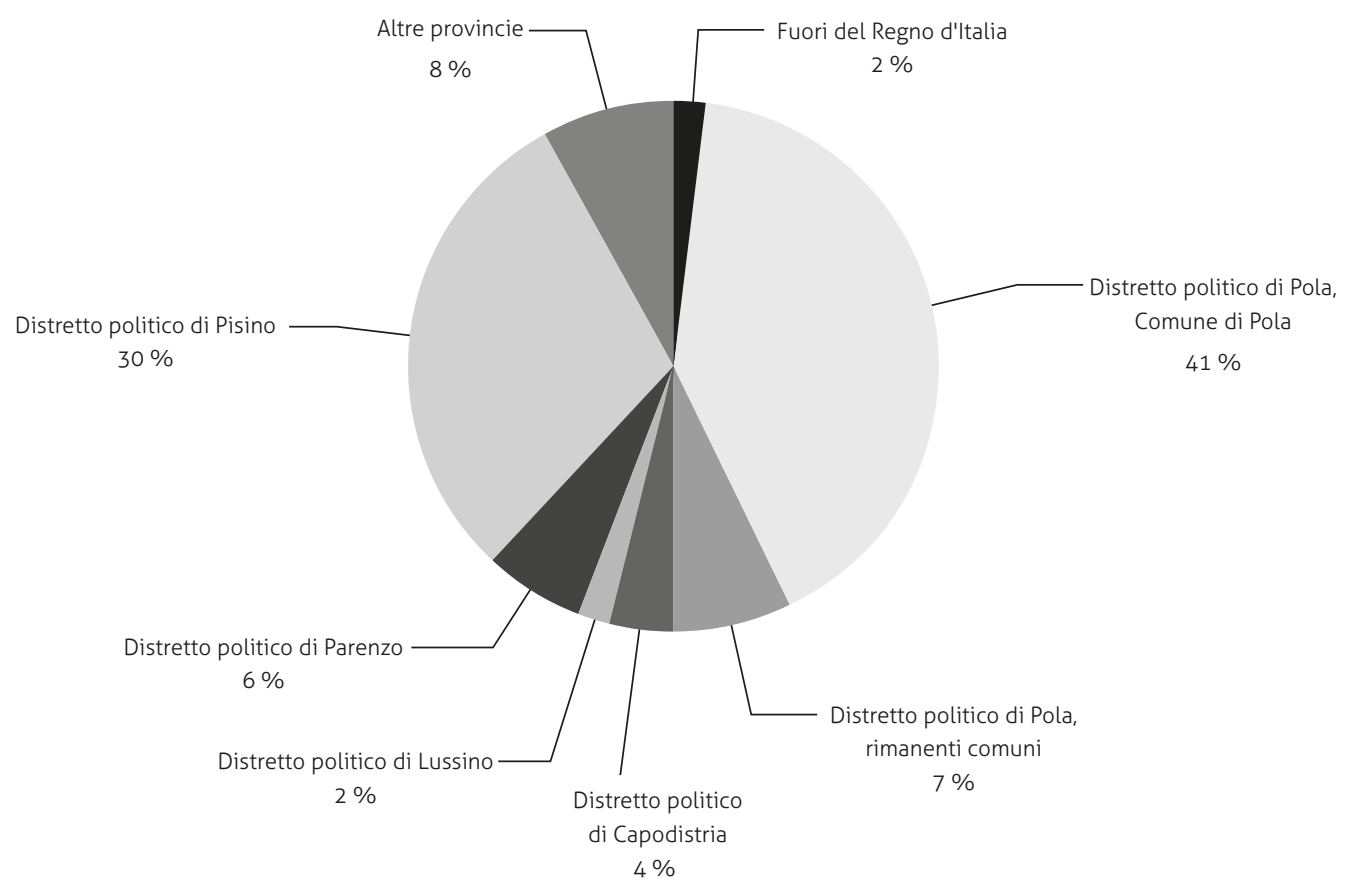

Grafico 4. Luogo di nascita dei residenti nella città di Pola risultanti nei decreti pubblicati

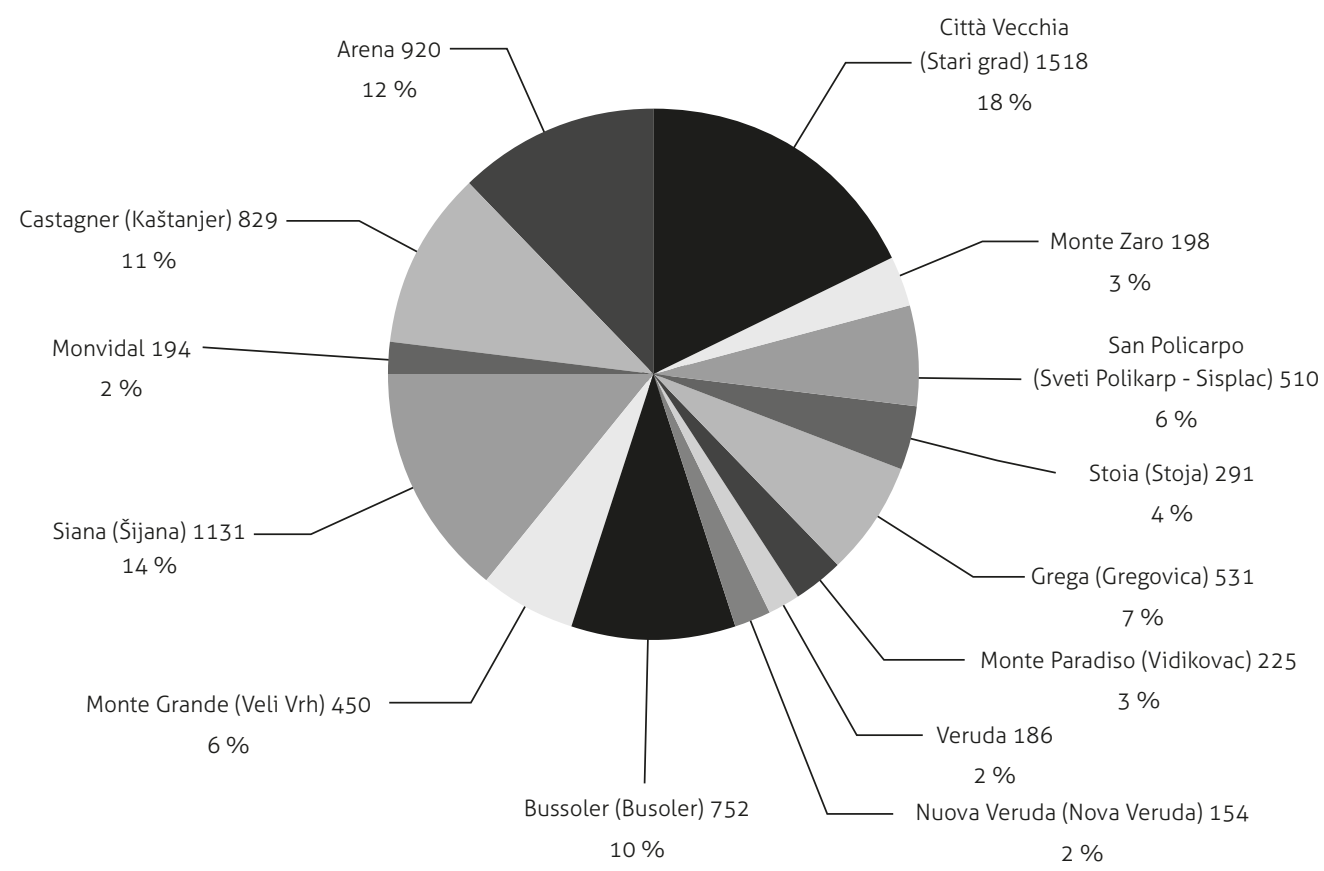

Grafico 5. Distribuzione per rione delle persone risultanti sui decreti con l'indirizzo indicato 


\subsection{Alcuni accenni su altri dati presenti nei decreti prefettizi della Provincia dell'Istria}

Pur non essendo negli scopi di questo lavoro, si impone un accenno al valore di questi documenti nel campo dell'onomastica. In primo luogo, essi ci hanno consentito l'identificazione di 2.983 cognomi unici per grafia, presenti sul territorio prima dell'italianizzazione, e la loro distribuzione geografica nel tempo. Sarebbe pertanto possibile studiare i flussi migratori nella regione.

In secondo luogo, questi documenti mostrano chiaramente come la ricchezza onomastica delle Province fosse problematica per il processo d'italianizzazione. Così che è possibile riscontrare divergenze fra i cognomi presenti nei decreti pubblicati e quelli nell'elenco ufficiale allestito dalla Prefettura di Pola. Queste riguardano sia i cognomi tenuti "a riassumere forma italiana" sia quelli sostitutivi.

Prendendo i cognomi inizianti con la lettera "A" (piccolissimo campione rispetto ad altre lettere come "B", "C", "S") notiamo che sulla G.U. non compaiono 24 cognomi presenti invece nell'elenco, mentre è vero il contrario per 5 cognomi (Alman, Antoninich, Anzur, Arnicevich e Augscheller). Questo non solo conferma l'ipotesi che non tutti i decreti emessi siano stati pubblicati facendo pensare all'adozione di procedure arbitrarire nel cambio dei cognomi, ma indica pure che il deterioramento del patrimonio onomastico sia ancor più ampio di quello desumibile dall'elenco pubblicato sul settimanale Istarska riječ.

Pure i 2.298 cognomi sostitutivi proposti dalla G.U. divergono dai cognomi sostitutivi presenti nell'elenco. Questo è dovuto soprattutto alle scelte fatte dai richiedenti, ma non solo. Prendendo a esempio il cognome Codacovich, presente su 18 decreti, notiamo che questo veniva cambiato d'ufficio in Codacco, come da elenco, ma pure in Codacci; fra i richiedenti si notano altri 5 diversi cognomi.

\section{Considerazioni finali}

La politica di snazionalizzazione costituisce solo una parte dell'ampio programma della politica avviata dallo Stato italiano nel primo dopoguerra contro le minoranze delle Nuove Province. Questa politica assume nel periodo fascista una forma nuova: "Per la prima volta, vengono impiegati contro le minoranze tutti gli strumenti di potere di uno Stato moderno" ${ }^{95}$. Lita- 
lianizzazione dei cognomi, elemento di questa politica, è testimoniata dai documenti la cui analisi è qui esposta. Allo scopo di definire numericamente questo "evento emblematico che cercò di occultare le tracce della diversità rappresentata da comunità e da culture potenzialmente non omologabili” sono stati elaborati tutti i 49.310 decreti prefettizi che, appoggiandosi su leggi e regi decreti approvati appositamente, pronunciavano il cambiamento di cognome di centinaia di migliaia di individui con la pubblicazione sul principale organo dello Stato, la Gazzetta Ufficiale del Regno d'Italia.

Per i documenti provenienti dalle prefetture di tutte le province è stata fatta l'analisi numerica, l'analisi delle modalità di emissione e dei ritmi di pubblicazione. Il numero dei documenti e la loro vasta distribuzione territoriale legittimano l'uso del termine "genocidio culturale" ${ }^{57}$.

Onde meglio precisare i meccanismi e la portata del fenomeno vengono analizzati a fondo 16.815 documenti riguardanti la Provincia dell'Istria. Si inizia con il quadro nominale dei prefetti e viceprefetti della Prefettura di Pola che ne furono esecutori per passare in seguito all'analisi del loro operato. Si è potuto stabilire che i documenti da loro emessi convalidano l'italianizzazione forzata del cognome di 70.145 individui - ben oltre i 56.000 sin ora proposti ${ }^{58}$. In base al comune di residenza di ciascuno di essi si è allestita la distribuzione geografica dei cognomi coinvolti. Appare evidente una maggiore pressione sui comuni delle isole e su quelli costieri della penisola istriana. Si mette però in evidenza come anche il retroterra istriano, fortemente popolato da croati e sloveni, sia stato toccato dal cambiamento o con decreti mai pubblicati oppure ipso iure, senza alcun decreto prefettizio ${ }^{59}$. Viene inoltre abbozzata un'analisi separata per il capoluogo della Provincia.

Il materiale si presta a ulteriori analisi per tutte le province. Per la Provincia dell'Istria in particolare la base dati allestita nel corso di questa ricerca offre informazioni nel campo onomastico che andrebbero analizzate ulteriormente non solo per capire il periodo che produsse i documenti qui esaminati, ma anche per comprendere gli eventi successivi.

\footnotetext{
56 Tasso, "Fascismo e cognomi", 323.

57 Apih, Italia, fascismo e antifascismo, 129; Wörsdörfer, Il confine orientale, 111.

58 Čermelj, Sloveni e croati, 147; Apih, Italia, fascismo e antifascismo, 282.

59 Tasso, "Fascismo e cognomi”, 322, citando Merkù.
} 


\section{Riassunto}

L'italianizzazione forzata dei cognomi, parte del più ampio programma di snazionalizzazione delle minoranze nelle Nuove Province (Trento, Bolzano, Trieste, Istria, Gorizia, Fiume, Zara) avviato dallo Stato italiano nel primo dopoguerra, fu legalizzata per mezzo di leggi e decreti votati appositamente dal giovane governo fascista e implementata con l'istituzione, presso le prefetture di ogni provincia, di commissioni responsabili per la compilazione di elenchi contenenti i cognomi ritenuti non italiani e le loro rispettive forme sostitutive. L'iter, non sempre rispettato, prevedeva il cambiamento del cognome su domanda del capofamiglia (con l'affissione sul rispettivo albo comunale) oppure per imposizione della prefettura. Dopodiché, il cambiamento veniva pronunciato con un decreto del Prefetto della Provincia, notificato agli interessati, pubblicato nella Gazzetta Ufficiale del Regno d'Italia ed annotato nei registri dello stato civile.

La ricerca qui esposta ha considerato e analizzato numericamente tutti i decreti di cambiamento di cognome pubblicati nella Gazzetta Ufficiale. Poiché lo Stato italiano non sempre adempì all'obbligo di pubblicare tutti i decreti, il risultato ottenuto non può che essere incompleto. Nondimeno testimonia una vera e propria ossessione onomastica che coinvolse centinaia di migliaia di individui.

Infine, un'analisi più dettagliata dei decreti emessi dalla Prefettura di Pola dimostra quanta e quale fu la mobilitazione dell'apparato del potere che, dietro il dichiarato desiderio di "recupero dei cognomi originari italiani o latini", perpetrava quello che la storiografia definirà come "genocidio culturale" e "onomasticidio di Stato".

\section{Prefekturalne odredbe objavljene u Gazzetti Ufficiale del Regno d'Italia i prisilna talijanizacija prezimena u Novim pokrajinama s posebnim osvrtom na Istarsku pokrajinu}

\section{Sažetak}

Prisilna talijanizacija prezimena, kao dio širega programa denacionalizacije manjina u Novim pokrajinama (Trento, Bolzano, Trst, Istra, Gorica, Rijeka, Zadar) koji je talijanska država pokrenula nakon Prvoga svjetskog rata, legalizirana je zakonima i uredbama koje je mlada fašistička vlada posebno izglasala i implementirala kroz instituciju povjerenstava, nazočnih u prefekturi svake pokrajine i odgovornih za sastavljanje popisa svih prezimena koja nisu bila smatrana talijanskima te njihovih odgovarajućih zamjenskih oblika. Postupak, koji se nije uvijek poštivao, predviđao je promjenu prezimena na zahtjev glave obitelji (uz objavu na općinskoj oglasnoj ploči) ili bi ju nametnula prefektura. Nakon toga, promjena bi bila proglašena uredbom provincijskoga prefekta, zainteresirane strane bi bile obaviještene, a promjena bi se objavila u Gazzetti Ufficiale del Regno d'Italia i zapisala u matične knjige.

Istraživanje je uzelo u obzir i numerički analiziralo sve uredbe o promjeni prezimena objavljenih u Gazzetti Ufficiale. Budući da talijanska država nije uvijek objavljivala sve uredbe o promjeni prezimena, dobiveni su rezultati nepotpuni, no svejedno svjedoče o pravoj onomastičkoj opsesiji koja je zahvatila stotine tisuća pojedinaca. 
Na kraju, podrobnija analiza dekreta koje je izdala Prefektura u Puli pokazuje kolika i kakva je bila mobilizacija državnoga aparata koji je, pod krinkom izražene želje za „obnavljanjem izvornih talijanskih ili latinskih prezimena”, počinio ono što će historiografija zabilježiti kao „kulturni genocid” i „državni onomastički genocid”.

\section{Prefectorial decrees published by the Gazzetta Ufficiale del Regno d'Italia and the compulsory italianization of the surnames present in the Nuove Province with a focus on the Istrian province}

\section{Summary}

The denationalization plan of the minorities living in the Nuove Province (Trento, Bolzano, Trieste/Trst, Istria/Istra, Gorizia/Gorica, Fiume/Rijeka, Zara/Zadar) was started by the Italian State in the aftermath of World War I and comprised the compulsory Italianization of surnames. The latter process was legalized by means of laws and decrees passed by the new fascist government. In each provincial prefecture, committees were established to put together a register recording all surnames considered non-Italian along with their respective substitutive forms. The formal procedure, with which it was not always complied, required that a request for surname change either came from the head of the household (by posting it on the City Hall bulletin board) or from the prefecture by imposition. Afterwards, the change was declared valid with a decree signed by the Province Prefect, notified to the individual involved, published on the Gazzetta Ufficiale del Regno d'Italia, and recorded in the civil registry.

In the present work, we considered and numerically analyzed all decrees concerning a surname change and published in the Gazzetta Ufficiale. Since the Italian State not always complied with the obligation to publish all decrees, our results cannot be complete. Nonetheless, they attest to the sheer onomastic obsession that affected hundreds of thousands of individuals.

Lastly, a more detailed analysis of the decrees issued by the Pola/Pula Prefecture shows to what extent the dominant power was engaged in this process. Behind the stated desire to recover the original Italian and Latin surnames, the fascist regime was in fact perpetrating what historiography later called "cultural genocide" and "State onomasticide". 


\section{Allegato}

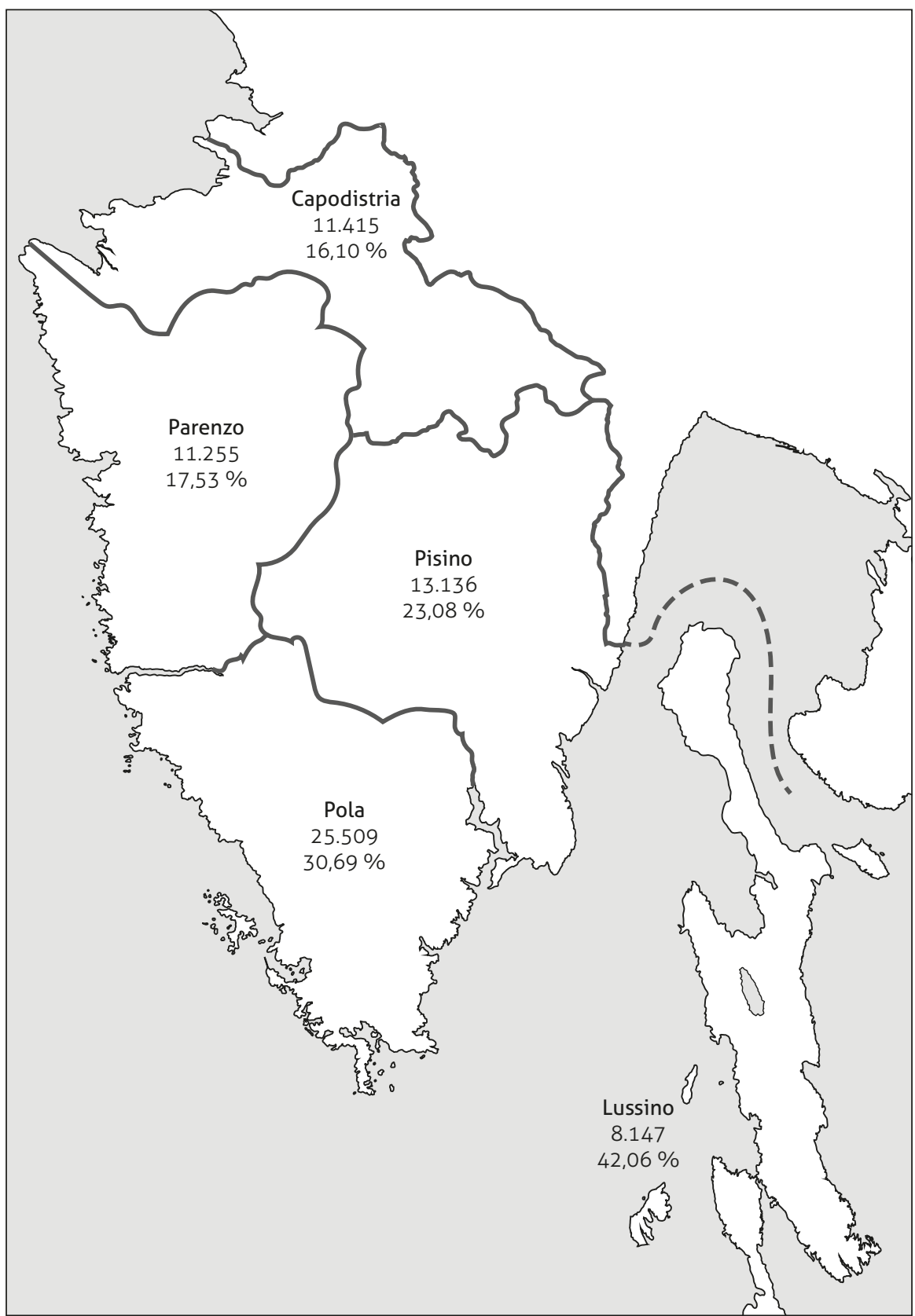

Mappa 1. Distribuzione geografica delle persone coinvolte per distretto politico di residenza e percentuale rispetto al numero di abitanti al 21 aprile 1936 